\title{
Nonlinear Theory for Coalescing Characteristics in Multiphase Whitham Modulation Theory
}

\author{
Thomas J. Bridges $^{1}$ (D) . Daniel J. Ratliff ${ }^{2}$
}

Received: 4 May 2020 / Accepted: 27 November 2020 / Published online: 29 December 2020

(C) The Author(s) 2020

\begin{abstract}
The multiphase Whitham modulation equations with $N$ phases have $2 N$ characteristics which may be of hyperbolic or elliptic type. In this paper, a nonlinear theory is developed for coalescence, where two characteristics change from hyperbolic to elliptic via collision. Firstly, a linear theory develops the structure of colliding characteristics involving the topological sign of characteristics and multiple Jordan chains, and secondly, a nonlinear modulation theory is developed for transitions. The nonlinear theory shows that coalescing characteristics morph the Whitham equations into an asymptotically valid geometric form of the two-way Boussinesq equation, that is, coalescing characteristics generate dispersion, nonlinearity and complex wave fields. For illustration, the theory is applied to coalescing characteristics associated with the modulation of two-phase travelling wave solutions of coupled nonlinear Schrödinger equations, highlighting how collisions can be identified and the relevant dispersive dynamics constructed.
\end{abstract}

Keywords Lagrangian · Averaging · Wavetrains · Jordan chains · Multisymplectic

Mathematics Subject Classification 37K58 · 70H33 · 35P30

\section{Introduction}

The theory of modulation, particularly Whitham modulation theory, takes the existing nonlinear waves, such as finite-amplitude periodic travelling waves, and provides a

Communicated by Peter Miller.

$凶$ Thomas J. Bridges

t.bridges@surrey.ac.uk

1 Department of Mathematics, University of Surrey, Guildford GU2 7XH, UK

2 Department of Mathematical Sciences, Loughborough University, Loughborough, Leicestershire LE11 3TU, UK 
framework for studying the dynamical implications of perturbing the basic properties of the nonlinear wave. In classical modulation, the properties of the basic state (wavenumber, frequency, mean flow) are allowed to depend on space and time, and partial differential equations (PDEs) are derived for these parameters. Study of these PDEs then provides information about the evolution of the basic state under perturbation.

Given a basic state, there are several strategies for deriving modulation PDEs (averaging the Lagrangian, averaging conservation laws, geometric optics ansatz, other ansätze). In all cases, the governing equations produced by Whitham modulation theory (WMT), for a simple one-phase periodic travelling wave, can be expressed in the canonical form

$$
q_{T}=\Omega_{X} \text { and } \frac{\partial}{\partial T} \mathscr{A}(\omega+\Omega, k+q)+\frac{\partial}{\partial X} \mathscr{B}(\omega+\Omega, k+q)=0 .
$$

They are a pair of nonlinear first-order PDEs for the two unknowns $\Omega(X, T)$, the modulation frequency, and $q(X, T)$, the modulation wavenumber. The parameters $(\omega, k)$ are representative of the wavetrain from which the Whitham modulation equations are obtained, and $X=\varepsilon x$ and $T=\varepsilon t$ are slow time and space scales. The first equation is called conservation of waves and the second is called conservation of wave action (Whitham 1974). When the governing equations are the Euler-Lagrange equations associated with a Lagrangian functional, the scalar-valued functions $\mathscr{A}$ and $\mathscr{B}$ are related via

$$
\mathscr{A}=\mathscr{L}_{\omega}, \quad \mathscr{B}=\mathscr{L}_{k}
$$

The function $\mathscr{L}(\omega, k)$ is obtained by averaging the Lagrangian evaluated on the periodic travelling wave with frequency $\omega$ and wavenumber $k$.

The pair of quasilinear first-order equation (1.1) can be classified based on their characteristics. The Whitham modulation equations (WMEs) can either be hyperbolic (real characteristics) or elliptic (complex characteristics) and the transition signals a change of stability of the underlying periodic waves (Whitham 1965, 1974; Bridges and Ratliff 2017, 2018). It is this change of type, and its generalization to multiphase wavetrains, and its nonlinear implications, that are the main themes of this paper.

To identify the structure of coalescing characteristics, first consider the one-phase case where only two characteristics exist and so coalescence is elementary. The linearization of the one-phase WMEs (1.1) about the basic state, represented by $(\omega, k)$, is

$$
q_{T}=\Omega_{X} \text { and } \mathscr{A}_{\omega} \Omega_{T}+\mathscr{A}_{k} q_{T}+\mathscr{B}_{\omega} \Omega_{X}+\mathscr{B}_{k} q_{X}=0,
$$

or, under the assumption $\mathscr{A}_{\omega} \neq 0$, they can be written in the standard hydrodynamical form,

$$
\left(\begin{array}{c}
q \\
\Omega
\end{array}\right)_{T}+\mathbf{F}(\omega, k)\left(\begin{array}{c}
q \\
\Omega
\end{array}\right)_{X}=\left(\begin{array}{l}
0 \\
0
\end{array}\right)
$$


with

$$
\mathbf{F}(\omega, k)=\frac{1}{\mathscr{A}_{\omega}}\left[\begin{array}{cc}
0 & -\mathscr{A}_{\omega} \\
\mathscr{B}_{k} & \mathscr{A}_{k}+\mathscr{B}_{\omega}
\end{array}\right]
$$

Here, $\mathscr{A}$ and $\mathscr{B}$ are evaluated at $\Omega=q=0$. The characteristics (eigenvalues of $\mathbf{F}$ ) are

$$
c^{ \pm}=\frac{\mathscr{A}_{k}+\mathscr{B}_{\omega}}{2 \mathscr{A}_{\omega}} \pm \frac{1}{\mathscr{A}_{\omega}} \sqrt{-\Delta_{L}},
$$

where

$$
\Delta_{L}=\mathscr{A}_{\omega} \mathscr{B}_{k}-\mathscr{A}_{k} \mathscr{B}_{\omega}=\operatorname{det}\left[\begin{array}{cc}
\mathscr{L}_{\omega \omega} & \mathscr{L}_{\omega k} \\
\mathscr{L}_{k \omega} & \mathscr{L}_{k k}
\end{array}\right],
$$

using (1.2) in the latter equality. The sign of the determinant $\Delta_{L}$, called the Lighthill determinant (Lighthill 1967), signals whether the characteristics are real or complex,

$$
\begin{aligned}
& \Delta_{L}<0 \Longrightarrow \text { hyperbolic WMEs } \\
& \Delta_{L}>0 \Longrightarrow \text { elliptic WMEs }
\end{aligned}
$$

At the transition, when $\Delta_{L}=0$, the two characteristics are equal, the Whitham modulation equations degenerate, and a new modulation strategy is needed. In Bridges and Ratliff (2017), a nonlinear modulation theory is developed for the above case within the WMEs in the case of one-phase wavetrains. It is valid near the transition from hyperbolic to elliptic, showing that the WMEs (1.1) are replaced by

$$
q_{T}=\Omega_{X} \text { and } \mathscr{A}_{\omega} \Omega_{T}+\kappa q q_{X}+\mathscr{K} q_{X X X}=0,
$$

where $T=\varepsilon^{2} t, X=\varepsilon\left(x-c_{g} t\right)$, and $c_{g}$ is a nonlinear group velocity at the transition. The coefficients $\mathscr{A}_{\omega}$ and $\kappa$ are obtained from derivatives of the components of conservation of wave action, and the dispersion coefficient $\mathscr{K}$ arises due to a symplectic Jordan chain argument. Differentiating the second equation of (1.8) with respect to $X$ and using the first equation reveals that it is a variant of the two-way Boussinesq equation for $q$,

$$
\mathscr{A}_{\omega} q_{T T}+\left(\frac{1}{2} \kappa q^{2}+\mathscr{K} q_{X X}\right)_{X X}=0 .
$$

The coefficients in (1.8) and (1.9) are universal in the same sense that the Whitham equations are universal - they follow from abstract properties of a Lagrangian. Extension of the derivation of (1.8) to two space dimensions and time appears in Bridges and Ratliff (2018). The emergence of Eq. (1.9) shows that coalescing characteristics generate nonlinearity, dispersion and wave fields of greater complexity. The complexity is due to the wide range of known localized, multi-pulse, quasiperiodic, and extreme value solutions of the two-way Boussinesq equation. 
In order to generalize this nonlinear theory for coalescing characteristics to the case of multiphase wavetrains, several new results are needed. The first results on nongeneric Whitham modulation theory, in the multiphase case, considered the case when the generic WMEs have a single or double zero characteristic. In Ratliff and Bridges (2016), it was shown that a zero characteristic in WMT leads, under re-modulation, to Korteweg-de Vries (KdV) dynamics on a longer time scale. In Ratliff (2018a), a double zero characteristic is re-modulated leading to dynamics governed by a two-way Boussinesq equation.

The motivation for re-modulation, in both cases, is that a zero characteristic in the generic WMEs suggests no dynamics, but in fact the dynamics is moved to a slower time scale. The time scale $t \sim \varepsilon^{-1}$, in generic WMT, is replaced by $t \sim \varepsilon^{-2}$ in Ratliff (2018a) and is replaced by $t \sim \varepsilon^{-3}$ in Ratliff and Bridges (2016). The double zero characteristic is a special case of coalescing characteristics. It does not require the theory of coalescing characteristics with nonzero speeds such as the sign characteristic, and it is codimension two.

In this paper, we are interested in the nonlinear theory near coalescing characteristics with nonzero speed. This case is codimension one and so more likely to occur in applications, and it requires the theory of the sign characteristic to track collisions of characteristics. It was discovered in Bridges and Ratliff (2019) that every characteristic in the Whitham theory carries a topological sign, and this sign is an important diagnostic as only coalescing characteristics with opposite sign can change type from hyperbolic to elliptic. In addition, several facets of the linear theory, such as intertwining Jordan chains, that generate the coefficient $\mathscr{K}$, bring in new challenges. For the nonlinear theory, we find that the form of the two-way Boussinesq equation (1.9) carries over to the case of coalescing characteristics with nonzero speed, but there is a discrepancy between the fact that (1.9) is scalar-valued but the WMEs in the multiphase case have $2 N$ equations. Hence, a secondary reduction of the nonlinear equations is required. Showing that the coefficients are universal is also an order of magnitude more difficult in this case.

The mathematics of how characteristics coalesce and change type is addressed as follows. Firstly consider the one-phase case. The change of type of the characteristics signals an instability of the basic state, and this linear instability is made apparent by taking the normal mode ansatz

$$
\left(\begin{array}{c}
q(X, T) \\
\Omega(X, T)
\end{array}\right)=\operatorname{Re}\left\{\left(\begin{array}{c}
\widehat{q} \\
\widehat{\Omega}
\end{array}\right) \mathrm{e}^{\lambda T+\mathrm{i} \nu X}\right\},
$$

and substituting into (1.3) to obtain

$$
\lambda= \pm \mathrm{i} c^{ \pm} \nu= \pm \mathrm{i} v\left(\frac{\mathscr{A}_{k}+\mathscr{B}_{\omega}}{2 \mathscr{A}_{\omega}} \pm \frac{1}{\mathscr{A}_{\omega}} \sqrt{-\Delta_{L}}\right) .
$$

There are four $\lambda$-eigenvalues for fixed $v \neq 0$ since $\widehat{q}$ and $\widehat{\Omega}$ are complex-valued. An unstable exponent $(\operatorname{Re}(\lambda)>0)$ with modulation wave number $v$ exists precisely when $\Delta_{L}>0$. As $\Delta_{L}$ changes sign, the eigenvalues (1.11) change from four purelyimaginary eigenvalues to a complex quartet as shown schematically in Fig. 1. This 


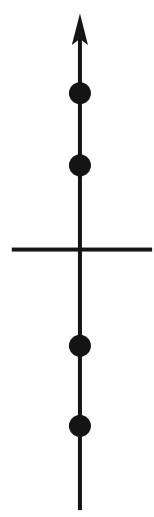

$\Delta_{L}<0$

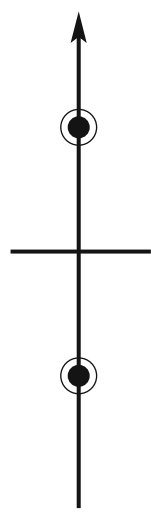

$\Delta_{L}=0$

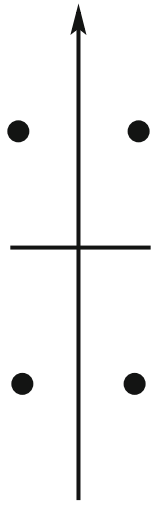

$\Delta_{L}>0$

Fig. 1 Collision of purely imaginary eigenvalues in the Whitham equations

type of stability transition is familiar from the theory of linear Hamiltonian systems, as it is precisely the Hamiltonian Hopf bifurcation (van der Meer 1985), and in that setting the collision and resulting instability occur since the eigenvalues have opposite Krein signature (Howard 2013; van der Meer 1985). However, as shown in Bridges and Ratliff (2019), there is no obvious symplectic structure in the Whitham theory, and it is the sign characteristic of Hermitian matrix pencils that is operational here. The sign characteristic has a central role in the theory of Hermitian matrix pencils relative to an indefinite metric (see Gohberg et al. 2005 for a history and references).

The Hermitian matrix pencil structure of (1.3) is evoked by multiplying the conservation of waves by $\mathscr{A}_{\omega}$, assuming $\mathscr{A}_{\omega} \neq 0$, and combining the two equations in (1.3) as

$$
\left[\begin{array}{cc}
0 & \mathscr{A}_{\omega} \\
\mathscr{A}_{\omega} & \mathscr{A}_{k}+\mathscr{B}_{\omega}
\end{array}\right]\left(\begin{array}{l}
\Omega \\
q
\end{array}\right)_{T}+\left[\begin{array}{cc}
-\mathscr{A}_{\omega} & 0 \\
0 & \mathscr{B}_{k}
\end{array}\right]\left(\begin{array}{l}
\Omega \\
q
\end{array}\right)_{X}=\left(\begin{array}{l}
0 \\
0
\end{array}\right) .
$$

The two coefficient matrices are symmetric. Now the modified normal mode ansatz

$$
\left(\begin{array}{l}
\Omega(X, T) \\
q(X, T)
\end{array}\right)=\operatorname{Re}\left\{\left(\begin{array}{l}
\widehat{\Omega} \\
\widehat{q}
\end{array}\right) \mathrm{e}^{\mathrm{i} v(X+c T)}\right\},
$$

generates the following Hermitian matrix eigenvalue problem

$$
\left(\left[\begin{array}{cc}
-\mathscr{A}_{\omega} & 0 \\
0 & \mathscr{B}_{k}
\end{array}\right]+c\left[\begin{array}{cc}
0 & \mathscr{A}_{\omega} \\
\mathscr{A}_{\omega} & \mathscr{A}_{k}+\mathscr{B}_{\omega}
\end{array}\right]\right)\left(\begin{array}{l}
\widehat{\Omega} \\
\widehat{q}
\end{array}\right)=\left(\begin{array}{l}
0 \\
0
\end{array}\right) .
$$

The theory of Hermitian matrix pencils shows that each eigenvalue of (1.13) has a sign characteristic and a necessary condition for instability is that eigenvalues coalesce and have opposite sign characteristic (Bridges and Ratliff 2019). In the one phase case, with just two characteristics, the sign characteristic is less interesting, and indeed trivial. In the multiphase case, with many characteristics, the coalescence of characteristics 
may or may not lead to instability, and so the sign characteristic becomes an essential diagnostic tool. The principal case of interest in this paper is when all the characteristics are real, with only one pair, having opposite sign, undergoing a transition to instability.

The generalization of the dispersionless WMEs (1.1) to the multiphase case is

$$
\mathbf{q}_{T}=\boldsymbol{\Omega}_{X} \text { and } \frac{\partial}{\partial T} \mathbf{A}(\boldsymbol{\omega}+\boldsymbol{\Omega}, \mathbf{k}+\mathbf{q})+\frac{\partial}{\partial X} \mathbf{B}(\boldsymbol{\omega}+\boldsymbol{\Omega}, \mathbf{k}+\mathbf{q})=0,
$$

where $\boldsymbol{\omega}, \mathbf{k} \in \mathbb{R}^{N}$ are given parameters representative of the basic state, and $\mathbf{q}, \boldsymbol{\Omega} \in \mathbb{R}^{N}$ are the vector-valued unknowns, the modulation wavenumber and frequency, which depend on $T=\varepsilon t$ and $X=\varepsilon x$. This general form of the WMEs (1.14) covers not only multiply-periodic waves, but quasiperiodic structures, and WMEs on pseudo-phases which model mean flow (this latter case is discussed in Sect. 3.1). When the governing equations are the Euler-Lagrange equation associated with a Lagrangian functional, the mappings $\mathbf{A}$ and $\mathbf{B}$ are again variations of the average Lagrangian $\mathscr{L}(\boldsymbol{\omega}, \mathbf{k})$ with the properties,

$$
\mathbf{A}(\boldsymbol{\omega}+\mathbf{\Omega}, \mathbf{k}+\mathbf{q})=\mathrm{D}_{\omega} \mathscr{L}(\boldsymbol{\omega}+\boldsymbol{\Omega}, \mathbf{k}+\mathbf{q})
$$

and

$$
\mathbf{B}(\boldsymbol{\omega}+\boldsymbol{\Omega}, \mathbf{k}+\mathbf{q})=\mathrm{D}_{\mathbf{k}} \mathscr{L}(\boldsymbol{\omega}+\boldsymbol{\Omega}, \mathbf{k}+\mathbf{q}) .
$$

Cross-differentiating shows that the Jacobians satisfy

$$
\mathrm{D}_{\mathbf{k}} \mathbf{A}=\left(\mathrm{D}_{\omega} \mathbf{B}\right)^{T}
$$

This symmetry will be important for generalizing the Hermitian property of (1.13) to the multiphase case.

Given a smooth averaged Lagrangian $\mathscr{L}$, the pair of Eq. (1.14) is a closed firstorder system of PDEs for $\boldsymbol{\Omega}$ and $\mathbf{q}$ with up to $2 N$ characteristics. This formulation of vector-valued WMEs was introduced in Ratliff (2018a, 2017a). However, multiphase Whitham modulation theory has a rich history. Multiphase WMEs were first introduced and studied by Ablowitz and Benney (1970), in the context of scalar nonlinear wave equations, where the appearance of small divisors was noted. For integrable systems small divisors disappear: multiphase averaging and the Whitham equations are robust and rigorous, and a general theory can be obtained (e.g. Flashka et al. 1980 and its citation trail). WHITHAM [Whitham (1974), Sect. 14.7] includes potential variables as additional phases ("pseudo-phases" which are included in the theory here; see Sect. 3.1) and generates a form of multiphase modulation and applies it to wavemean flow interaction of Stokes water waves (Whitham 1967). Willebrand (1975) takes multiphase modulation theory to a new level by deriving the $N$-phase WMEs, for Stokes wave solutions of the water wave problem, with $N$ arbitrary and he takes the limit $N \rightarrow \infty$. This theory is formal and the series are divergent and have small divisors, but the leading order terms are instructive (see comments on this later in 
Sect. 9.7). The theory of Willebrand (1975) is now used in ocean wave forecasting (e.g. Chapter 9 of Olbers et al. 2012).

On the other hand, when the system is not integrable, but there is an $N$-fold symmetry, a theory for conservation of wave action can be developed without small divisors and smoothly varying $N$-phase wavetrains. This strategy is implemented in Ratliff (2018a, 2017a), where multiphase wavetrains are characterized as relative equilibria with smooth dependence on parameters.

Going back to the abstract multiphase WMEs (1.14), with the symmetry property (1.17) and the gradient properties (1.15), the linearization of (1.14) can be cast into the form of a Hermitian matrix pencil,

$$
\left[\left[\begin{array}{cc}
-\mathrm{D}_{\omega} \mathbf{A} & 0 \\
0 & \mathrm{D}_{\mathbf{k}} \mathbf{B}
\end{array}\right]+c\left[\begin{array}{cc}
0 & \mathrm{D}_{\omega} \mathbf{A} \\
\mathrm{D}_{\omega} \mathbf{A} & \mathrm{D}_{\mathbf{k}} \mathbf{A}+\mathrm{D}_{\omega} \mathbf{B}
\end{array}\right]\right]\left(\begin{array}{l}
\widehat{\boldsymbol{\Omega}} \\
\widehat{\mathbf{q}}
\end{array}\right)=\left(\begin{array}{l}
\mathbf{0} \\
\mathbf{0}
\end{array}\right),
$$

assuming that $\mathrm{D}_{\omega} \mathbf{A}$ is invertible. The Jacobians $\mathrm{D}_{\omega} \mathbf{A}, \mathrm{D}_{\mathbf{k}} \mathbf{B}, \mathrm{D}_{\omega} \mathbf{B}$, and $\mathrm{D}_{\mathbf{k}} \mathbf{A}$, are $N \times N$ matrices, with the first two symmetric and the latter two are the matrix transpose of one another. The $2 N \times 2 N$ linear eigenvalue problem (1.18) can be reduced, by eliminating $\widehat{\boldsymbol{\Omega}}$,

$$
\widehat{\mathbf{\Omega}}=c \widehat{\mathbf{q}}, \quad\left(\text { assuming } \operatorname{det}\left[\mathrm{D}_{\omega} \mathbf{A}\right] \neq 0\right),
$$

to an $N \times N$ quadratic Hermitian matrix pencil,

$$
\mathbf{E}(c) \widehat{\mathbf{q}}:=\left[\mathrm{D}_{\omega} \mathbf{A} c^{2}+c\left(\mathrm{D}_{\mathbf{k}} \mathbf{A}+\mathrm{D}_{\omega} \mathbf{B}\right)+\mathrm{D}_{\mathbf{k}} \mathbf{B}\right] \widehat{\mathbf{q}}=\mathbf{0} .
$$

A parallel theory can be developed for the sign characteristic in this context (Gohberg et al. 1980; Mehrmann et al. 2016; Tisseur and Meerbergen 2001). Suppose $c_{0}$ is a simple real eigenvalue satisfying $\operatorname{det}\left[\mathbf{E}\left(c_{0}\right)\right]=0$ with eigenvector $\zeta$, so that

$$
\mathbf{E}\left(c_{0}\right) \zeta=0
$$

Then, the sign characteristic of $c_{0}$ is

$$
S\left(c_{0}\right)=\operatorname{sign}\left(\left\langle\zeta, \mathbf{E}^{\prime}\left(c_{0}\right) \zeta\right\rangle\right)
$$

where $\langle\cdot, \cdot\rangle$ is an inner product on $\mathbb{R}^{N}$, and the prime denotes differentiation with respect to $c$. A discussion of the history and various formulations of the sign characteristic is given in Bridges and Ratliff (2019). The sign characteristic is invariant under congruence transformation, $\mathbf{E}\left(c_{0}\right) \mapsto \mathbf{P}^{T} \mathbf{E}\left(c_{0}\right) \mathbf{P}$, for any invertible $\mathbf{P}$ (Gohberg et al. 1980). The quadratic formulation (1.20), rather than its linearization (1.18), turns out to be the most efficient in applications and arises naturally in the modulation theory.

Starting with the quadratic Hermitian matrix pencil (1.20) a theory for the sign characteristic in the context of Whitham modulation theory is developed by Bridges and Ratliff (2019). The $2 N$ characteristics of the linearized problem satisfy

$$
\Delta(c):=\operatorname{det}[\mathbf{E}(c)]=0 .
$$


Double non-semisimple characteristics, which characterize coalescence, and define the nonlinear group velocity $c_{g}$, satisfy

$$
\Delta\left(c_{g}\right)=\Delta^{\prime}\left(c_{g}\right)=0 \text { but } \Delta^{\prime \prime}\left(c_{g}\right) \neq 0,
$$

with a single geometric eigenvector

$$
\mathbf{E}\left(c_{g}\right) \zeta=0
$$

and generalized eigenvector

$$
\mathbf{E}\left(c_{g}\right) \boldsymbol{\gamma}=-\mathbf{E}^{\prime}\left(c_{g}\right) \zeta
$$

Solvability of (1.26) defines $c_{g}$. All these properties follow from the structure of the linear operator $\mathbf{E}(c)$ with $c \in \mathbb{R}$ and are studied in Bridges and Ratliff (2019) and the details required here are recorded in Sect. 4 and their role in the Jordan chain theory is developed in Sect. 6.

When multiphase modulation is introduced for the nonlinear problem, with an appropriate scaling, the vector-valued conservation of wave action (1.14) will be morphed into another form with dispersion. It will however still have dimension $N$, so a further reduction is necessary in order to obtain a generalization of (1.9). The strategy is to split $\mathbb{R}^{N}=\operatorname{span}\{\zeta\} \oplus \mathbb{R}^{N-1}$. The geometric eigenvector $\zeta$, defined in (1.21), provides a preferred direction in $\mathbf{q}$-wavenumber space associated with the coalescence. This preferred direction is an essential part of the nonlinear modulation theory. It provides a projection operator so that the vector-valued conservation of wave action (1.14) can be reduced to a scalar equation, and this scalar equation, which also requires a rescaling of the slow variables, and extension of the analysis to fifth order in $\varepsilon$, is a geometric form of the scalar-valued two-way Boussinesq equation

$$
\mu U_{T T}+\frac{1}{2} \kappa\left(U^{2}\right)_{X X}+\mathscr{K} U_{X X X X}=0
$$

where $\mu$ and $\kappa$ are determined by the geometry of the averaged Lagrangian $\mathscr{L}(\omega, \mathbf{k})$ and $\mathscr{K}$ is determined by a twisted Jordan chain argument.

The geometry of $\mathscr{L}(\boldsymbol{\omega}, \mathbf{k})$ is discussed in Sect. 2.2. The most remarkable outcome of the geometry is that the coefficient $\kappa$ in (1.27) has the simple formula

$$
\kappa:=\left.\frac{d^{3}}{d s^{3}} \mathscr{L}\left(\boldsymbol{\omega}+s c_{g} \boldsymbol{\zeta}, \mathbf{k}+s \zeta\right)\right|_{s=0} .
$$

The coefficient $\mu$ is determined by a Jordan chain associated with the linear operator $\mathbf{E}\left(c_{g}\right)$ in (1.25). Indeed, $\mu \neq 0$ is the condition required for termination of the Jordan chain $(\zeta, \gamma)$ in (1.25)-(1.26). A different Jordan chain, associated with the linearization of the Euler-Lagrange equations [denoted $\mathbf{L}$ in (2.9)], determines the dispersion coefficient $\mathscr{K}$. This latter Jordan chain argument is similar to the case of multiphase modulation associated with zero characteristics in Ratliff (2018a, 2017a); Ratliff and 
Bridges (2016) but here the Jordan chain intertwines two different chains generated by $\mathbf{L}$. The nonlinear modulation theory where (1.28) arises naturally and feeds into the emergence of (1.27) is developed in Sect. 5.

The starting point for the theory is a general class of nonlinear PDEs generated by a Lagrangian, and this class is introduced in Sect. 2. Given a basic multiphase wavetrain $\widehat{Z}(\boldsymbol{\theta}, \boldsymbol{\omega}, \mathbf{k})$, with phase $\boldsymbol{\theta}=\mathbf{k} x+\boldsymbol{\omega} t+\boldsymbol{\theta}_{0}$ and vector-valued frequency and wavenumber $\boldsymbol{\omega}, \mathbf{k}$, satisfying the Euler-Lagrange equations, the dispersionless vectorvalued WMEs (1.14) are derived by modulating the basic state with a geometric optics scaling (Ratliff 2017a, 2018a; Bridges and Ratliff 2019). The appropriate modulation ansatz is

$$
Z(x, t)=\widehat{Z}\left(\boldsymbol{\theta}+\varepsilon^{-1} \boldsymbol{\phi}, \boldsymbol{\omega}+\boldsymbol{\Omega}, \mathbf{k}+\mathbf{q}\right)+\varepsilon W\left(\boldsymbol{\theta}+\varepsilon^{-1} \boldsymbol{\phi}, X, T, \varepsilon\right),
$$

where $\phi, \omega$ and $\mathbf{q}$, depending on $T=\varepsilon t$ and $X=\varepsilon x$, are the modulated phase, frequency and wavenumber, and $W$ is a remainder. Substitution of (1.29) into the Euler-Lagrange equation and solvability requires $\mathbf{q}$ and $\boldsymbol{\Omega}$ to satisfy (1.14) to leading order (Ratliff 2017a, 2018a).

When two characteristics, of opposite sign characteristic, coalesce and transition to instability, the geometric optics modulation ansatz

$$
\boldsymbol{\theta} \mapsto \boldsymbol{\theta}+\varepsilon^{-1} \boldsymbol{\phi}, \quad \omega \mapsto \omega+\boldsymbol{\Omega}, \quad \mathbf{k} \mapsto \mathbf{k}+\mathbf{q},
$$

(with $T=\varepsilon t$ and $X=\varepsilon x$ ) in (1.29) must be replaced. The altered form utilized is

$$
\boldsymbol{\theta} \mapsto \boldsymbol{\theta}+\varepsilon \boldsymbol{\Phi}, \quad \mathbf{k} \mapsto \mathbf{k}+\varepsilon^{2} \boldsymbol{\Phi}_{X}, \quad \boldsymbol{\omega} \mapsto \boldsymbol{\omega}+\varepsilon^{2} c_{g} \boldsymbol{\Phi}_{X}+\varepsilon^{3} \boldsymbol{\Phi}_{T},
$$

where $\boldsymbol{\Phi}$ is a function of the slow time and space variables,

$$
X=\varepsilon\left(x+c_{g} t\right), \quad T=\varepsilon^{2} t,
$$

with $c_{g}$ determined as part of the analysis, and $\varepsilon$ measuring the distance in $(\boldsymbol{\omega}, \mathbf{k})$-space from the singularity (1.24). The new ansatz at coalescence is

$$
Z(x, t)=\widehat{Z}\left(\boldsymbol{\theta}+\varepsilon \boldsymbol{\Phi}, \boldsymbol{\omega}+\varepsilon^{2} c_{g} \boldsymbol{\Phi}_{X}+\varepsilon^{3} \boldsymbol{\Phi}_{T}, \mathbf{k}+\varepsilon^{2} \boldsymbol{\Phi}_{X}\right)+\varepsilon^{3} W(\boldsymbol{\theta}, X, T, \varepsilon) .
$$

Finer detail on the ansatz, including definitions of $\mathbf{q}$ and $\boldsymbol{\Omega}$ and their relation to $\boldsymbol{\Phi}_{X}$ and $\boldsymbol{\Phi}_{T}$ is given in Sect. 5. Substitution of this ansatz into the governing Euler-Lagrange equations, expanding everything in powers of $\varepsilon$, and setting order by order to zero, results, by imposing a solvability condition, in a vector-valued two-way Boussinesq equation induced by conservation of wave action. The projection operator, defined using $\operatorname{Ker}\left(\mathbf{E}\left(c_{g}\right)\right)$, is then implemented to split the conservation of wave action into two parts, one generating the two-way Boussinesq equation (1.27) with the complementary part carrying over to higher order. 
The paper has four parts: the Lagrangian (geometry, analysis, and Euler-Lagrange equation), the linear theory (for both operators $\mathbf{E}(c)$ and $\mathbf{L}$ ), the nonlinear modulation analysis [implementing the ansatz (1.32)], and an illustrative example.

In Sect. 2 a class of Lagrangian functionals and a class of basic states is introduced. In Sect. 2.2 the geometry of the mapping $(\boldsymbol{\omega}, \mathbf{k}) \mapsto \mathscr{L}(\boldsymbol{\omega}, \mathbf{k})$, where $\mathscr{L}(\boldsymbol{\omega}, \mathbf{k})$ is the Lagrangian evaluated on a basic state, is studied. Remarkably, many of the features of the linear problem as well as the nonlinear modulation are determined by the geometry of this scalar-valued function. We end this discussion by reviewing the Whitham modulation theory from a geometric perspective in Sect. 3 to demonstrate how the characteristics and their coalescence may be formulated using these notions, as discussed in Sect. 4. In Sect. 3.1, we show how the theory of pseudo-phases, and their relation with mean flow, fits into the theory of this paper.

The linear theory has two parts: the structure of the linear operator $\mathbf{E}(c)$ in (1.20), including the Jordan chain theory in the setting of the quadratic eigenvalue problem (1.20). This theory is developed in Sects. 2.2 and 6, and appeals to the theory of sign characteristic for Hermitian matrix pencils developed in Bridges and Ratliff (2019). The second part of the linear theory is the linearization of the Euler-Lagrange equation, which is needed to develop a secondary Jordan chain needed for constructing the dispersion coefficient $\mathscr{K}$ and the nonlinear modulation theory, and this theory is developed in Sect. 6.

The nonlinear theory is developed in Sect. 5. Although the ansatz (1.32) with (1.30) is new, once the ansatz is identified the strategy is similar to our previous papers, particularly Bridges and Ratliff (2017) and Ratliff and Bridges (2016), and so only the key new features are highlighted. The theory is illustrated by application to the two-phase travelling wave solutions of a class of coupled nonlinear Schrödinger (CNLS) equations. In Bridges and Ratliff (2019), it was shown that these travelling wave solutions have coalescing characteristics with transition to instability. Here, the theory is applied to show the emergence of a geometric two-way Boussinesq equation at these singularities. Potential generalizations are discussed in the concluding remarks section.

\section{The Lagrangian and Governing Equations}

The theory is built on a general class of Lagrangian functionals

$$
\mathcal{L}(V)=\int_{t_{1}}^{t_{2}} \int_{x_{1}}^{x_{2}} L\left(V, V_{t}, V_{x}, \ldots\right) \mathrm{d} x \mathrm{~d} t,
$$

where $V(x, t)$ is a vector-valued smooth field defined on the rectangle $\left[x_{1}, x_{2}\right] \times\left[t_{1}, t_{2}\right]$. The lower dots indicate that the Lagrangian may also depend on higher derivatives of $V$, and the subsequent theory can be adapted for these cases. Potential variables are included by letting some components of $V$ appear in $L(\cdot)$ with derivatives only (see discussion in Sect. 3.1 of pseudo-phases associated with potential variables).

Normally a non-degeneracy condition on derivatives of $L$ with respect to $V_{t}$ and $V_{x}$ is assumed, but here these conditions are circumvented by assuming up front that 
the Lagrangian has been transformed to standard multisymplectic form

$$
\mathcal{L}(Z)=\int_{t_{1}}^{t_{2}} \int_{x_{1}}^{x_{2}}\left[\frac{1}{2}\left\langle\mathbf{M} Z_{t}, Z\right\rangle+\frac{1}{2}\left\langle\mathbf{J} Z_{x}, Z\right\rangle-S(Z)\right] \mathrm{d} x \mathrm{~d} t,
$$

where $Z \in \mathbb{R}^{n}, \mathbf{M}$ and $\mathbf{J}$ are skew-symmetric matrices, $S: \mathbb{R}^{n} \rightarrow \mathbb{R}$ is a given smooth function, and $\langle\cdot, \cdot\rangle$ is a standard inner product on $\mathbb{R}^{n}$. For definiteness, $n$ is taken to be even and

$$
\operatorname{det}[\mathbf{J}+c \mathbf{M}] \neq 0, \quad \forall c \in \mathscr{C} \subset \mathbb{R}
$$

where $\mathscr{C}$ is some open set of real numbers. Examples with $n=4$ for $\mathbf{M}$ and $\mathbf{J}$ include the dispersive shallow water equations (Bridges 2017, 2013),

$$
\mathbf{M}=\left(\begin{array}{cccc}
0 & -1 & 0 & 0 \\
1 & 0 & 0 & 0 \\
0 & 0 & 0 & 0 \\
0 & 0 & 0 & 0
\end{array}\right), \quad \text { and } \mathbf{J}=\left(\begin{array}{cccc}
0 & 0 & -1 & 0 \\
0 & 0 & 0 & -1 \\
1 & 0 & 0 & 0 \\
0 & 1 & 0 & 0
\end{array}\right)
$$

which has $\mathscr{C}=\mathbb{R}$, and the coupled-mode equation (also massive-Thirring equation),

$$
\mathbf{M}=\left(\begin{array}{cccc}
0 & -1 & 0 & 0 \\
1 & 0 & 0 & 0 \\
0 & 0 & 0 & 1 \\
0 & 0 & -1 & 0
\end{array}\right) \quad \text { and } \mathbf{J}=\left(\begin{array}{cccc}
0 & 0 & 1 & 0 \\
0 & 0 & 0 & -1 \\
-1 & 0 & 0 & 0 \\
0 & 1 & 0 & 0
\end{array}\right)
$$

with an appropriate choice of $S(Z)$ in both cases. The latter case has $\mathscr{C}=\mathbb{R} \backslash\{ \pm 1\}$, since $\operatorname{det}[\mathbf{J}+c \mathbf{M}]=\left(c^{2}-1\right)^{2}$. These examples and others can be found in Bridges and Derks (2001), Bridges et al. (2010), Bridges (2013, 2017).

The Euler-Lagrange equation associated with (2.1) is

$$
\mathbf{M} Z_{t}+\mathbf{J} Z_{x}=\nabla S(Z), \quad Z \in \mathbb{R}^{n}
$$

The theoretical developments to follow are based on this abstract form of the EulerLagrange equation, with $\mathbf{M}, \mathbf{J}$ general skew-symmetric matrices satisfying (2.2), and $n$ even with $n \geq 2 N$.

\subsection{Symmetry, Relative Equilibria and the Basic State}

The easiest way to generate smooth families of multiphase wavetrains is to consider a Lagrangian that is invariant under the action of a Lie group. Here, and henceforth it is assumed that the Lie group is abelian, and some combination of $S^{1}$ (associated with periodic wavetrains), and affine translations. Affine translations, which are associated 
with pseudo-phases, are captured in the formulation (2.3) by

$$
\left\langle\mathbf{e}_{j}, \nabla S(Z)\right\rangle=0, \quad j=1, \ldots, P,
$$

where $P$ is the dimension of the affine symmetry group, and $\mathbf{e}_{j} \in \mathbb{R}^{n}$ are the generators. The theory will be developed for the case of the $N$-torus, which is appropriate for periodic $N$-phase wavetrains, as the affine translation group is much simpler and the necessary changes will be recorded when needed.

Assume that (2.3) is equivariant with respect to an $N$-torus, $\mathbb{T}^{N}=\left(S^{1}\right)^{N}$, with matrix representation $G_{\theta}($ an $n \times n$ orthogonal matrix $)$ and $\theta=\left(\theta_{1}, \ldots, \theta_{N}\right)$. The infinitesimal generators are

$$
\mathrm{g}_{j}(Z):=\left.\frac{\partial}{\partial \theta_{j}} G_{\theta} Z\right|_{\theta=0}, \quad j=1, \ldots, N
$$

Since $G_{\theta}$ is orthogonal the action of $g_{j}$ on $Z$ is a skew-symmetric matrix. Equivariance of (2.3) then follows from the requirements

$$
G_{\theta} \mathbf{M}=\mathbf{M} G_{\theta}, \quad G_{\theta} \mathbf{J}=\mathbf{J} G_{\theta}, \quad \text { and } \quad S\left(G_{\theta} Z\right)=S(Z), \quad \forall G_{\theta} \in \mathbb{T}^{N}
$$

The basic state, namely the solution that will be modulated, is taken to be a family of periodic $N$-phase wavetrains of the form

$$
Z(x, t)=\widehat{Z}(\boldsymbol{\theta}, \mathbf{k}, \boldsymbol{\omega}), \quad \boldsymbol{\theta}=\mathbf{k} x+\boldsymbol{\omega} t+\boldsymbol{\theta}^{(0)},
$$

with the basic state (2.6) $2 \pi$-periodic in each component of $\boldsymbol{\theta}, \boldsymbol{\theta}^{(0)} \in \mathbb{R}^{N}$ a constant, and

$$
\boldsymbol{\theta}=\left(\begin{array}{c}
\theta_{1} \\
\vdots \\
\theta_{N}
\end{array}\right), \quad \mathbf{k}=\left(\begin{array}{c}
k_{1} \\
\vdots \\
k_{N}
\end{array}\right), \quad \boldsymbol{\omega}=\left(\begin{array}{c}
\omega_{1} \\
\vdots \\
\omega_{N}
\end{array}\right)
$$

Substitution of $\widehat{Z}$ into (2.3) admits the governing equation for the $N$-phase wavetrain

$$
\sum_{j=1}^{N}\left(\omega_{j} \mathbf{M}+k_{j} \mathbf{J}\right) \partial_{\theta_{i}} \widehat{Z}=\nabla S(\widehat{Z})
$$

In the absence of symmetry, solutions of this problem may encounter small divisors. The advantage of the $\mathbb{T}^{N}$-symmetry of (2.3) is that multiphase wavetrains are smooth functions with no small divisors: the wavetrain is a multiparameter family of relative equilibria. The relative equilibrium structure of the basic state (2.6) then gives

$$
\widehat{Z}(\boldsymbol{\theta}, \boldsymbol{\omega}, \mathbf{k})=G_{\boldsymbol{\theta}} \widehat{\mathbf{z}}(\boldsymbol{\omega}, \mathbf{k}) \quad \text { with } \quad \widehat{Z}_{\theta_{j}}=G_{\theta} \mathrm{g}_{j}(\widehat{\mathbf{z}}), \quad j=1, \ldots, N .
$$


All the dynamics is in the group action. The vector $\widehat{\mathbf{z}}(\boldsymbol{\omega}, \mathbf{k})$ can be thought of as the reference point along the group orbit, and satisfies

$$
\sum_{j=1}^{N}\left(\omega_{j} \mathbf{M}+k_{j} \mathbf{J}\right) g_{j}(\widehat{\mathbf{z}})=\nabla S(\widehat{\mathbf{z}})
$$

\subsubsection{Linearization About a Multiphase Wavetrain}

Associated with (2.7) is the linear operator

$$
\mathbf{L} V=\mathrm{D}^{2} S(\widehat{Z}) V-\sum_{j=1}^{N}\left(\omega_{j} \mathbf{M}+k_{j} \mathbf{J}\right) \partial_{\theta_{j}} V .
$$

This operator is formally self-adjoint with respect to the inner product

$$
\langle\langle\cdot, \cdot\rangle\rangle=\left(\frac{1}{2 \pi}\right)^{N} \int_{0}^{2 \pi} \cdots \int_{0}^{2 \pi}\langle\cdot, \cdot\rangle \mathrm{d} \theta_{1} \cdots \mathrm{d} \theta_{N}:=\int_{\mathbb{T}^{N}}\langle\cdot, \cdot\rangle \mathrm{d} \boldsymbol{\theta} .
$$

Differentiation of (2.7) with respect to each $\theta_{i}$ and each of the four parameters $k_{i}, \omega_{i}$ leads to the equations

$$
\begin{aligned}
\mathbf{L} \widehat{Z}_{\theta_{i}} & =0 \\
\mathbf{L} \widehat{Z}_{k_{i}} & =\mathbf{J} \widehat{Z}_{\theta_{i}}, \\
\mathbf{L} \widehat{Z}_{\omega_{i}} & =\mathbf{M} \widehat{Z}_{\theta_{i}}, \quad i=1, \ldots, N .
\end{aligned}
$$

The first of these equations highlights the fact that the kernel of $\mathbf{L}$ is at least $N$ dimensional, and in this paper, it is assumed no larger, so that

$$
\operatorname{Ker}(\mathbf{L})=\operatorname{span}\left\{\widehat{Z}_{\theta_{1}}, \ldots, \widehat{Z}_{\theta_{N}}\right\}
$$

The other equations in (2.11) will become significant when the Jordan chain theory in a moving frame is developed. The assumption (2.12) along with the formal selfadjointness of $\mathbf{L}$ give the solvability conditions for an expression $F$ to lie within the range of $\mathbf{L}$ as

$$
\mathbf{L} W=F \quad \Leftrightarrow \quad\left\langle\left\langle\widehat{Z}_{\theta_{1}}, F\right\rangle\right\rangle=\cdots=\left\langle\left\langle\widehat{Z}_{\theta_{N}}, F\right\rangle\right\rangle=0 .
$$

\subsubsection{Multisymplectic Noether Theory}

In the Lagrangian setting, the symmetry induces conservation laws via Noether theory. Transforming to a multisymplectic formulation then induces multisymplectic Noether theory which relates the structure operators $\mathbf{J}$ and $\mathbf{M}$ to the components of the induced conservation laws. Although these conservation laws may have other physical significance, they play the role of conservation of wave action in the Whitham theory and so the components will be called wave action and wave action flux. 
There is a conservation law associated with each phase of the wavetrain, and multisymplectic Noether theory implies the existence of functions $A_{j}, B_{j}$ satisfying

$$
\mathbf{M g}_{j}(Z)=\nabla A_{j}(Z), \quad \mathbf{J g}_{j}(Z)=\nabla B_{j}(Z), \quad j=1, \ldots, N
$$

and so

$$
A_{j}(x, t)=\int_{\mathbb{T}^{N}}\left\langle\mathbf{M g}_{j} Z, Z\right\rangle \mathrm{d} \boldsymbol{\theta}, \quad B_{j}(x, t)=\int_{\mathbb{T}^{N}}\left\langle\mathbf{J g}_{j} Z, Z\right\rangle \mathrm{d} \boldsymbol{\theta},
$$

where $Z(x, t, \boldsymbol{\theta})$ is a function of $(x, t)$ and the phases $\boldsymbol{\theta}=\left(\theta_{1}, \ldots, \theta_{N}\right)$ which are here interpreted as ensemble parameters. Direct calculation verifies that the conservation laws are

$$
\partial_{t} A_{j}+\partial_{x} B_{j}=0, \quad j=1, \ldots, N
$$

whenever $Z$ satisfies (2.3).

The components of the conservation laws can also be deduced directly from the averaged Lagrangian. The Lagrangian (2.1), evaluated on the $N$-phase wavetrain and averaged, is

$$
\mathscr{L}(\boldsymbol{\omega}, \mathbf{k})=\int_{\mathbb{T}^{N}}\left[\sum_{j=1}^{N}\left[\frac{1}{2} \omega_{j}\left\langle\widehat{Z}, \mathbf{M} \widehat{Z}_{\theta_{j}}\right\rangle+\frac{1}{2} k_{j}\left\langle\widehat{Z}, \mathbf{J} \widehat{Z}_{\theta_{j}}\right\rangle\right]-S(\widehat{Z})\right] \mathrm{d} \boldsymbol{\theta}
$$

The wave action vector evaluated on the wavetrain is

$$
\mathbf{A}(\boldsymbol{\omega}, \mathbf{k})=\left(\begin{array}{c}
\mathscr{A}_{1} \\
\vdots \\
\mathscr{A}_{N}
\end{array}\right):=\mathrm{D}_{\omega} \mathscr{L}=\left(\begin{array}{c}
\mathscr{L}_{\omega_{1}} \\
\vdots \\
\mathscr{L}_{\omega_{N}}
\end{array}\right)=\frac{1}{2}\left(\begin{array}{c}
\left\langle\left\langle\mathbf{M} \widehat{Z}_{\theta_{1}}, \widehat{Z}\right\rangle\right\rangle \\
\vdots \\
\left\langle\left\langle\mathbf{M} \widehat{Z}_{\theta_{N}}, \widehat{Z}\right\rangle\right\rangle
\end{array}\right)
$$

and the wave action flux vector is

$$
\mathbf{B}(\boldsymbol{\omega}, \mathbf{k})=\left(\begin{array}{c}
\mathscr{B}_{1} \\
\vdots \\
\mathscr{B}_{N}
\end{array}\right):=\mathrm{D}_{\mathbf{k}} \mathscr{L}=\left(\begin{array}{c}
\mathscr{L}_{k_{1}} \\
\vdots \\
\mathscr{L}_{k_{N}}
\end{array}\right)=\frac{1}{2}\left(\begin{array}{c}
\left\langle\left\langle\mathbf{J} \widehat{Z}_{\theta_{1}}, \widehat{Z}\right\rangle\right\rangle \\
\vdots \\
\left\langle\left\langle\mathbf{J} \widehat{Z}_{\theta_{N}}, \widehat{Z}\right\rangle\right\rangle
\end{array}\right) .
$$

By definition, we have the following

$$
\begin{aligned}
& \mathrm{D}_{\omega} \mathbf{A}=\left(\begin{array}{ccc}
\partial_{k_{1}} \mathscr{A}_{1} & \cdots & \partial_{k_{N}} \mathscr{A}_{1} \\
\vdots & \ddots & \vdots \\
\partial_{k_{1}} \mathscr{A}_{N} & \cdots & \partial_{k_{N}} \mathscr{A}_{N}
\end{array}\right) \\
& \mathrm{D}_{\mathbf{k}} \mathbf{A}=\left(\begin{array}{ccc}
\partial_{k_{1}} \mathscr{A}_{1} & \cdots & \partial_{k_{N}} \mathscr{A}_{1} \\
\vdots & \ddots & \vdots \\
\partial_{k_{1}} \mathscr{A}_{N} & \cdots & \partial_{k_{N}} \mathscr{A}_{N}
\end{array}\right)=\mathrm{D}_{\omega} \mathbf{B}^{T}, \quad \text { and } \quad \mathrm{D}_{\mathbf{k}} \mathbf{B}=\left(\begin{array}{ccc}
\partial_{k_{1}} \mathscr{B}_{1} & \cdots & \partial_{k_{N}} \mathscr{B}_{1} \\
\vdots & \ddots & \vdots \\
\partial_{k_{1}} \mathscr{B}_{N} & \cdots & \partial_{k_{N}} \mathscr{B}_{N}
\end{array}\right) \text {. }
\end{aligned}
$$


The entries of these tensors are related to solutions via

$$
\begin{aligned}
\partial_{\omega_{j}} \mathscr{A}_{i} & =\left\langle\left\langle\mathbf{M} \widehat{Z}_{\theta_{i}}, \widehat{Z}_{\omega_{j}}\right\rangle\right\rangle \\
\partial_{k_{j}} \mathscr{A}_{i} & =\left\langle\left\langle\mathbf{M} \widehat{Z}_{\theta_{i}}, \widehat{Z}_{k_{j}}\right\rangle,\right. \\
\partial_{k_{j}} \mathscr{B}_{i} & =\left\langle\left\langle\mathbf{J} \widehat{Z}_{\theta_{i}}, \widehat{Z}_{k_{j}}\right\rangle\right\rangle \\
\partial_{k_{j} k_{m}} \mathscr{B}_{i} & =\left\langle\left\langle\mathbf{J} \widehat{Z}_{\theta_{i} k_{m}}, \widehat{Z}_{k_{j}}\right\rangle\right\rangle+\left\langle\left\langle\mathbf{J} \widehat{Z}_{\theta_{i}}, \widehat{Z}_{k_{j} k_{m}}\right\rangle\right\rangle, \quad i, j, m=1, \ldots, N .
\end{aligned}
$$

The definition of the wave action and wave action flux in terms of derivatives of the averaged Lagrangian induces symmetry of the Jacobians,

$$
\partial_{k_{i}} \mathscr{B}_{j}=\left\langle\left\langle\mathbf{J} \widehat{Z}_{\theta_{j}}, \widehat{Z}_{k_{i}}\right\rangle\right\rangle=\left\langle\left\langle\mathbf{L} \widehat{Z}_{k_{j}}, \widehat{Z}_{k_{i}}\right\rangle\right\rangle=\left\langle\left\langle\widehat{Z}_{k_{j}}, \mathbf{L} \widehat{Z}_{k_{i}}\right\rangle\right\rangle=\left\langle\left\langle\widehat{Z}_{k_{j}}, \mathbf{J} \widehat{Z}_{\theta_{i}}\right\rangle\right\rangle=\partial_{k_{j}} \mathscr{B}_{i}
$$

and

$$
\partial_{k_{j}} \mathscr{A}_{i}=\left\langle\left\langle\mathbf{M} \widehat{Z}_{\theta_{i}}, \widehat{Z}_{k_{i}}\right\rangle\right\rangle=\left\langle\left\langle\widehat{Z}_{\omega_{i}}, \mathbf{J} \widehat{Z}_{k_{j}}\right\rangle=\partial_{\omega_{i}} \mathscr{B}_{j}, \quad i, j=1, \ldots, N\right.
$$

The key property in both (2.17) and (2.18) is that the left-hand side is in terms of the functions of $(\boldsymbol{\omega}, \mathbf{k})$ only and the right-hand side is expressed in terms of the properties of the Euler-Lagrange equation (2.3), namely through the structure matrices $\mathbf{J}$ and $\mathbf{M}$. It is this connection that is the essence of multisymplectic Noether theory, and it feeds into the nonlinear modulation theory.

\subsection{Geometry of the Averaged Lagrangian}

Many of the properties needed in the modulation theory can be deduced from the abstract mapping

$$
(\omega, \mathbf{k}) \mapsto \mathscr{L}(\omega, \mathbf{k})
$$

where $\mathscr{L}: \mathbb{R}^{N} \times \mathbb{R}^{N} \rightarrow \mathbb{R}$ is the averaged Lagrangian (2.16) and is assumed to be a smooth function.

The wave action and wave action flux emerge from $\mathscr{L}$ via

$$
\left.\frac{d}{d s} \mathscr{L}(\boldsymbol{\omega}+s \mathbf{u}, \mathbf{k}+s \mathbf{v})\right|_{s=0}=\langle\mathbf{A}(\boldsymbol{\omega}, \mathbf{k}), \mathbf{u}\rangle+\langle\mathbf{B}(\boldsymbol{\omega}, \mathbf{k}), \mathbf{v}\rangle, \quad \text { for any } \mathbf{u}, \mathbf{v} \in \mathbb{R}^{N}
$$

where $\langle\cdot, \cdot \cdot\rangle$ is an inner product on $\mathbb{R}^{N}$. The second derivative can be used to generate the linear operator $\mathbf{E}(c)$. First set $\mathbf{u}=c \mathbf{v}$ in the above expression and look at the 
derivative

$$
\begin{aligned}
\left.\frac{d^{2}}{d s^{2}} \mathscr{L}(\omega+s c \mathbf{v}, \mathbf{k}+s \mathbf{v})\right|_{s=0} & =\left.\frac{d}{d s}\langle\mathbf{A}(\boldsymbol{\omega}+s c \mathbf{v}, \mathbf{k}+s \mathbf{v}), c \mathbf{v}\rangle\right|_{s=0} \\
& \quad+\left.\frac{d}{d s}\langle\mathbf{B}(\omega+s \mathbf{v}, \mathbf{k}+s \mathbf{v}), \mathbf{v}\rangle\right|_{s=0} \\
= & \left\langle\mathrm{D}_{\omega} \mathbf{A}(\omega, \mathbf{k}) c \mathbf{v}, c \mathbf{v}\right\rangle+\left\langle\mathrm{D}_{\mathbf{k}} \mathbf{A}(\omega, \mathbf{k}) \mathbf{v}, c \mathbf{v}\right\rangle \\
& +\left\langle\mathrm{D}_{\omega} \mathbf{B}(\boldsymbol{\omega}, \mathbf{k}) \mathbf{v}, \mathbf{v}\right\rangle+\left\langle\mathrm{D}_{\mathbf{k}} \mathbf{B}(\boldsymbol{\omega}, \mathbf{k}) \mathbf{v}, \mathbf{v}\right\rangle \\
= & \left\langle\left(\mathrm{D}_{\omega} \mathbf{A} c^{2}+\left(\mathrm{D}_{\mathbf{k}} \mathbf{A}+\mathrm{D}_{\omega} \mathbf{B}\right) c+\mathrm{D}_{\mathbf{k}} \mathbf{B}\right) \mathbf{v}, \mathbf{v}\right\rangle \\
= & \langle\mathbf{E}(c) \mathbf{v}, \mathbf{v}\rangle, \quad \text { for any } \mathbf{v} \in \mathbb{R}^{N} .
\end{aligned}
$$

Hence,

$$
\mathbf{E}(c) \mathbf{v}=\left.\frac{d}{\mathrm{~d} s}[c \mathbf{A}(\omega+c s \mathbf{v}, \mathbf{k}+s \mathbf{v})+\mathbf{B}(\omega+c s \mathbf{v}, \mathbf{k}+s \mathbf{v})]\right|_{s=0}
$$

The most remarkable result following from derivatives of $\mathscr{L}$ is the expression for $\kappa$, the coefficient of nonlinearity in the emergent two-way Boussinesq equation (1.27). Introduce the one parameter path in $\mathscr{L}(\boldsymbol{\omega}, \mathbf{k})$,

$$
F(s)=\mathscr{L}\left(\boldsymbol{\omega}+s c_{g} \boldsymbol{\zeta}, \mathbf{k}+s \zeta\right)
$$

with $c_{g}$ here considered as fixed, and $\zeta \in \operatorname{Ker}\left(\mathbf{E}\left(c_{g}\right)\right)$. Then, differentiating and using (1.15) and (1.16) gives

$$
\begin{aligned}
F^{\prime}(s)= & \left\langle\mathbf{A}\left(\omega+s c_{g} \zeta, \mathbf{k}+s \zeta\right), c_{g} \zeta\right\rangle+\left\langle\mathbf{B}\left(\boldsymbol{\omega}+s c_{g} \boldsymbol{\zeta}, \mathbf{k}+s \zeta\right), \zeta\right\rangle \\
F^{\prime \prime}(s)= & \left\langle\mathrm{D}_{\boldsymbol{\omega}} \mathbf{A}\left(\boldsymbol{\omega}+s c_{g} \zeta, \mathbf{k}+s \zeta\right) c_{g} \zeta, c_{g} \zeta\right\rangle+\left\langle\mathrm{D}_{\mathbf{k}} \mathbf{A}\left(\boldsymbol{\omega}+s c_{g} \zeta, \mathbf{k}+s \zeta\right) \zeta, c_{g} \zeta\right\rangle \\
& +\left\langle\mathrm{D}_{\boldsymbol{\omega}} \mathbf{B}\left(\boldsymbol{\omega}+s c_{g} \zeta, \mathbf{k}+s \zeta\right) c_{g} \zeta, \zeta\right\rangle+\left\langle\mathbf{B}_{\mathbf{k}}\left(\boldsymbol{\omega}+s c_{g} \zeta, \mathbf{k}+s \zeta\right) \zeta, \zeta\right\rangle
\end{aligned}
$$

Evaluating $F^{\prime \prime}(0)$,

$$
F^{\prime \prime}(0)=\left\langle c_{g}^{2} \mathrm{D}_{\omega} \mathbf{A} \zeta, \zeta\right\rangle+\left\langle c_{g} \mathrm{D}_{\mathbf{k}} \mathbf{A} \zeta, \zeta\right\rangle+\left\langle c_{g} \mathrm{D}_{\omega} \mathbf{B} \zeta, \zeta\right\rangle+\left\langle\mathbf{B}_{\mathbf{k}} \zeta, \zeta\right\rangle=\left\langle\mathbf{E}\left(c_{g}\right) \zeta, \zeta\right\rangle=0 .
$$

However, it is the third derivative of $F(s)$ that is of most interest. The formula for $F^{\prime \prime}(s)$ suggests that $F^{\prime \prime \prime}(s)$ is a derivative of a path through the linear operator $\mathbf{E}\left(c_{g}\right)$, considered as a function of $(\boldsymbol{\omega}, \mathbf{k})$ with $c_{g}$ fixed. Differentiating,

$$
\begin{aligned}
F^{\prime \prime \prime}(0) & :=\left.\frac{d^{3}}{d s^{3}} \mathscr{L}\left(\omega+s c_{g} \zeta, \mathbf{k}+s \zeta\right)\right|_{s=0} \\
& =\left\langle\zeta,\left(\mathrm{D}_{\mathbf{k}}^{2} \mathbf{B}+c_{g}\left(2 \mathrm{D}_{\mathbf{k}} \mathrm{D}_{\omega} \mathbf{B}+\mathrm{D}_{\mathbf{k}}^{2} \mathbf{A}\right)+c_{g}^{2}\left(2 \mathrm{D}_{\mathbf{k}} \mathrm{D}_{\omega} \mathbf{A}+\mathrm{D}_{\omega}^{2} \mathbf{B}\right)+c_{g}^{3} \mathrm{D}_{\omega}^{2} \mathbf{A}\right)(\zeta, \zeta)\right\rangle \\
& :=\kappa .
\end{aligned}
$$

At this point, this expression is just a formula, but the inner product in the second row will emerge naturally in the modulation theory in a solvability condition, giving it relevance as the coefficient of the nonlinear term in the emergent modulation equation. 
In a similar way, the coefficient $\mu$ in (1.27) can also be represented in terms of derivatives of $\mathscr{L}$ as in

$$
\mu=\left.\frac{d^{2}}{d s^{2}} \mathscr{L}(\boldsymbol{\omega}+s \zeta, \mathbf{k})\right|_{s=0}+\left.\frac{d^{2}}{\mathrm{~d} s^{2}} \mathscr{L}\left(\boldsymbol{\omega}+s c_{g} \boldsymbol{\gamma}, \mathbf{k}+s \boldsymbol{\gamma}\right)\right|_{s=0} .
$$

However, a more interesting characterization of $\mu$ is as a termination condition for the Jordan chain $(\boldsymbol{\zeta}, \boldsymbol{\gamma})$ in (1.25)-(1.26) [see Eqs. (4.9) and (4.10)].

\section{Generic Multiphase Whitham Equations}

In this section, a construction of the generic (distinct characteristics) multiphase WMEs is sketched from the paper's geometric perspective. It serves as a touchstone for the modifications needed for the non-generic (coalescing characteristics) case, and the generic theory is needed to define $c_{g}$, the frame speed at coalescence.

Given the basic state $\widehat{Z}$ in (2.6), with $N$ phase variables $\theta_{j}$, the generic WMEs are obtained using the geometric optics ansatz (Ratliff 2017a, 2018a),

$$
Z(x, t)=\widehat{Z}\left(\boldsymbol{\theta}+\varepsilon^{-1} \boldsymbol{\phi}, \boldsymbol{\omega}+\boldsymbol{\Omega}, \mathbf{k}+\mathbf{q}\right)+\varepsilon W\left(\boldsymbol{\theta}+\varepsilon^{-1} \boldsymbol{\phi}, X, T, \varepsilon\right)
$$

with $X=\varepsilon x$ and $T=\varepsilon t$, and the $N$-dimensional vectors $\boldsymbol{\phi}, \boldsymbol{\Omega}$, and $\mathbf{q}$ depending on $X, T$ and satisfying conservation of waves $\mathbf{q}_{T}=\boldsymbol{\Omega}_{X}$. Expand all terms in a Taylor series, e.g. $W=W_{1}+\mathcal{O}(\varepsilon)$, substitute into (2.3) and solve the equations at each order of $\varepsilon$. At zeroth order the governing equations for the basic wave $\widehat{Z}$ are recovered and at first order an equation for $W_{1}$ is obtained

$$
\mathbf{L} W_{1}=\sum_{j=1}^{N}\left[\partial_{T} \Omega_{j} \mathbf{M} \widehat{Z}_{\omega_{j}}+\partial_{T} q_{j} \mathbf{M} \widehat{Z}_{k_{j}}+\partial_{X} \Omega_{j} \mathbf{J} \widehat{Z}_{\omega_{j}}+\partial_{X} q_{j} \mathbf{J} \widehat{Z}_{k_{j}}\right]
$$

Applying the solvability conditions (2.13), and using the connection between the resulting expressions and the components of the conservation law (2.19a)-(2.19c), i.e.

$$
\begin{aligned}
& \left\langle\left\langle\widehat{Z}_{\theta_{i}}, \mathbf{M} \widehat{Z}_{\omega_{j}}\right\rangle\right\rangle=-\partial_{\omega_{j}} \mathscr{A}_{i},\left\langle\left\langle\widehat{Z}_{\theta_{i}}, \mathbf{J} \widehat{Z}_{k_{j}}\right\rangle\right\rangle=-\partial_{k_{j}} \mathscr{A}_{i}, \\
& \left\langle\left\langle\widehat{Z}_{\theta_{i}}, \mathbf{J} \widehat{Z}_{\omega_{j}}\right\rangle\right\rangle=-\partial_{k \omega_{j}} \mathscr{B}_{i},\left\langle\left\langle\widehat{Z}_{\theta_{i}}, \mathbf{J} \widehat{Z}_{k_{j}}\right\rangle=-\partial_{k_{j}} \mathscr{B}_{i}, i, j=1, \ldots, N,\right.
\end{aligned}
$$

then gives the generic WMEs,

$0=\sum_{j=1}^{N}\left[\partial_{T} \Omega_{j} \partial_{\omega_{j}} \mathscr{A}_{i}+\partial_{T} q_{j} \partial_{k_{1}} \mathscr{A}_{i}+\partial_{X} \Omega_{j} \partial_{\omega_{j}} \mathscr{B}_{i}+\partial_{X} q_{j} \partial_{k_{j}} \mathscr{B}_{i}\right], \quad i=1, \ldots, N$

Taking into account that $\widehat{Z}$ is a function of $\mathbf{k}+\mathbf{q}$ and $\boldsymbol{\omega}+\boldsymbol{\Omega}$, averaging over the phase eliminates the $\varepsilon^{-1} \boldsymbol{\phi}$ terms, and using the vector definition of wave action (2.17) and 
wave action flux (2.18), these two equations are the vector conservation equation

$$
\partial_{T} \mathbf{A}(\mathbf{k}+\mathbf{q}, \boldsymbol{\omega}+\boldsymbol{\Omega})+\partial_{X} \mathbf{B}(\mathbf{k}+\mathbf{q}, \boldsymbol{\omega}+\boldsymbol{\Omega})=0,
$$

which, when combined with conservation of waves and the symmetry condition

$$
\partial_{T} \mathbf{q}=\partial_{X} \boldsymbol{\Omega} \text { and } \mathrm{D}_{\mathbf{k}} \mathbf{A}=\left(\mathrm{D}_{\omega} \mathbf{B}\right)^{T},
$$

give the generic WMEs in vector form. Further details of the above derivation can be found in Ratliff (2017a, 2018a). A proof of validity of these multiphase WMEs, when the original equation is coupled NLS, covering both the cases of elliptic and hyperbolic characteristics, in the context of coupled nonlinear Schrödinger equations, is given in Bridges et al. (2020).

Consider the linearization of (3.2) and (3.3) at $(\boldsymbol{\omega}, \mathbf{k})$

$$
\mathrm{D}_{\omega} \mathbf{A} \boldsymbol{\Omega}_{T}+\mathrm{D}_{\mathbf{k}} \mathbf{A} \mathbf{q}_{T}+\mathrm{D}_{\omega} \mathbf{B} \boldsymbol{\Omega}_{X}+\mathrm{D}_{\mathbf{k}} \mathbf{B} \mathbf{q}_{X}=\mathbf{0} \text { and } \boldsymbol{\Omega}_{X}=\mathbf{q}_{T} .
$$

Characteristics about any state $(\boldsymbol{\omega}+\boldsymbol{\Omega}, \mathbf{k}+\mathbf{q})$ can be obtained the same way, but here the main interest is in characteristics in the neighbourhood of the basic state. Differentiating the first equation and using the second results in a second-order equation for q,

$$
\mathrm{D}_{\omega} \mathbf{A} \mathbf{q}_{T T}+\left(\mathrm{D}_{\mathbf{k}} \mathbf{A}+\mathrm{D}_{\omega} \mathbf{B}\right) \mathbf{q}_{T X}+\mathrm{D}_{\mathbf{k}} \mathbf{B} \mathbf{q}_{X X}=\mathbf{0} .
$$

With the normal mode ansatz

$$
(\boldsymbol{\Omega}, \mathbf{q})=(\widehat{\boldsymbol{\Omega}}, \widehat{\mathbf{q}}) \mathrm{e}^{\mathrm{i} \alpha(X+c T)},
$$

the second-order equation results in a quadratic equation for the characteristics,

$$
\mathbf{E}(c) \widehat{\mathbf{q}}:=\left[\mathrm{D}_{\omega} \mathbf{A} c^{2}+\left(\mathrm{D}_{\mathbf{k}} \mathbf{A}+\mathrm{D}_{\omega} \mathbf{B}\right) c+\mathrm{D}_{\mathbf{k}} \mathbf{B}\right] \widehat{\mathbf{q}}=\mathbf{0} .
$$

It is a Hermitian quadratic matrix polynomial, and there is an extensive literature on the properties of these matrices (e.g. Gohberg et al. 1980; Tisseur and Meerbergen 2001; Mehrmann et al. 2016 and references therein).

A key property that we will need is that a simple root, say $c_{0}$, has a "sign characteristic". A necessary condition for two characteristics to coalesce and transition from hyperbolic to elliptic is that they have opposite sign characteristic. A study of the sign characteristic in the context of the linearized multiphase WMEs is given in Bridges and Ratliff (2019), and the basics of the theory needed here is given in Sect. 4.

\subsection{Pseudo-Phases and Affine Symmetry}

The basic states considered in Sect. 2.1 are $2 \pi$-periodic in each phase (2.6). There are also pseudo-phases. In this section, it is shown that pseudo-phases can be treated the 
same as phases associated with periodic motion, noting only that no averaging over pseudo-phases is required. A group-theoretic interpretation of pseudo-phases is also given.

The concept of pseudo-phases was introduced by Whitham in his first papers on modulation (e.g. Whitham 1965, 1967) and discussed in more detail in Sect. 14.6 in Whitham (1974). The associated pseudo frequencies and pseudo wavenumbers play a role in mean flow.

Consider one of the most well-known examples of pseudo-phases; that is, mean flow in the water wave problem, using the simplified model,

$$
h_{t}+u h_{x}+h u_{x}=0 \text { and } u_{t}+u u_{x}+g h_{x}=\sigma h_{x x x},
$$

where $h(x, t)$ is the fluid depth, $u(x, t)$ is the horizontal velocity field, and $\sigma$ is a parameter. Let $u=\phi_{x}$, and then the Lagrangian variational principle that generates (3.6) is

$$
\delta \int_{t_{1}}^{t_{2}} \int_{x_{1}}^{x_{2}} \mathcal{L}\left(\phi_{t}, \phi_{x}, h_{t}, h_{x}, h\right) \mathrm{d} x \mathrm{~d} t=0,
$$

with

$$
\mathcal{L}\left(\phi_{t}, \phi_{x}, h_{t}, h_{x}, h\right)=h \phi_{t}+\frac{1}{2} h \phi_{x}^{2}+\frac{1}{2} g h^{2}+\frac{1}{2} \sigma h_{x}^{2}
$$

The key property is that the Lagrangian is invariant if a constant is added to $\phi$. This is an affine symmetry, the abstract group is the group of real numbers, and the action is

$$
s \cdot(h, \phi)=(h, \phi+s), \quad \forall s \in \mathbb{R} .
$$

This affine symmetry is reminiscent of "cyclic variables" in classical mechanics.

When we introduce a pseudo-phase associated with this group, $s=\beta x+\gamma t$, the pseudo-phase does not appear explicitly in the Lagrangian and so averaging is not required. However, we will see that a modulation equation is still generated of the same mathematical form. Let

$$
\phi=\beta x+\gamma t,
$$

and substitute into (3.7),

$$
\mathscr{L}(h, \beta):=\mathcal{L}(\gamma, \beta, 0,0, h)=\gamma h+\frac{1}{2} h \beta^{2}+\frac{1}{2} g h^{2}
$$

Although no averaging is required, Whitham modulation theory proceeds in the same way. Suppose $\beta(X, T)$ and $\gamma(X, T)$ are taken to depend on slow time and space 
variables. Then, the WMEs associated with the single pseudo-phase are

$$
\beta_{T}=\gamma_{X} \text { and } \frac{\partial}{\partial T}\left(\frac{\partial \mathscr{L}}{\partial \gamma}\right)+\frac{\partial}{\partial X}\left(\frac{\partial \mathscr{L}}{\partial \beta}\right)=0, \quad \text { and } \quad \frac{\partial \mathscr{L}}{\partial h}=0 .
$$

Differentiating $\mathscr{L}$ and substituting gives

$$
\beta_{T}=\gamma_{X} \text { and } \frac{\partial}{\partial T}(h)+\frac{\partial}{\partial X}(\beta h), \quad \text { and } \quad \gamma+\frac{1}{2} \beta^{2}+g h=0 .
$$

Substituting the third into the first, we get two equations

$$
\beta_{T}+\beta \beta_{X}+g h_{X}=0 \text { and } h_{T}+\beta h_{X}+h \beta_{X}=0 .
$$

The upshot here is twofold, the introduction of a pseudo-phase does not require averaging, and the resulting modulation equations are mathematically the same as the case of modulation of periodic waves.

Here, we have the added outcome that Whitham theory applied to the pseudo-phase (3.8) results in the classical shallow water equation (3.11), with $\beta(x, t)$ representing the horizontal velocity. If we add in a periodic phase as well, replacing (3.8) with

$$
\phi(x, t)=\beta x+\gamma t+\Phi(\theta), \quad \theta=k x+\omega t,
$$

where $\Phi$ is $2 \pi$-periodic, and substitute into the Lagrangian, then averaging would be required but only over the periodic phase. Modulating $\omega(X, T)$ and $k(X, T)$, leads to the WMEs associated with the periodic phase

$$
k_{T}=\omega_{X} \quad \text { and } \quad \frac{\partial}{\partial T}\left(\frac{\partial \mathscr{L}}{\partial \omega}\right)+\frac{\partial}{\partial X}\left(\frac{\partial \mathscr{L}}{\partial k}\right)=0, \quad \text { and } \quad \frac{\partial \mathscr{L}}{\partial E}=0
$$

where $E$ is representative of the amplitude. Combining (3.10) and (3.12) results in coupled multiphase modulation equations with the same mathematical form as if both phases were periodic. Indeed, this is precisely what was done in Whitham (1967).

By eliminating the amplitudes in (3.10) and (3.12) and relabelling $\gamma=\omega_{1}, \beta=k_{1}$, $\omega=\omega_{2}$, and $k=k_{2}$, the coupled multiphase modulation equations associated with pseudo-phase or phase take the same canonical form as (3.2) and (3.3). Hence, the theory of this paper for multiphase Whitham modulation theory, including the theory for coalescing characteristics, applies to both periodic phases and pseudo-phases. Henceforth, the results will be stated for the periodic case and can easily be adjusted for pseudo-phases.

\section{Defining Characteristics and Coalescence}

In this section, the algebraic structure of the quadratic Hermitian matrix pencil $\mathbf{E}(c)$ in (3.5) is discussed. Characteristics of the linearized WMEs (3.4) are the values of $c$ 
that are roots of the polynomial

$$
\Delta(c):=\operatorname{det}[\mathbf{E}(c)]=0 .
$$

When there are $N$-phases, this polynomial has degree $2 N$. The linear algebra of quadratic Hermitian matrix pencils can be found in Gohberg et al. (1980), Mehrmann et al. (2016), Tisseur and Meerbergen (2001) and references therein. Here, a theory for the sign characteristic of simple roots and the theory of double non-semisimple roots is required.

For definiteness, we assume that all the characteristics are hyperbolic and one pair transitions from hyperbolic to elliptic at some parameter value. It is not essential to the nonlinear theory for the other $2(N-1)$ characteristics to be hyperbolic at coalescence, although if they are not hyperbolic then the basic state is already unstable.

A characteristic is double when

$$
\Delta\left(c_{g}\right)=\Delta^{\prime}\left(c_{g}\right)=0 \text { and } \Delta^{\prime \prime}\left(c_{g}\right) \neq 0
$$

where $\Delta(c)$ is defined in (4.1). The value of $c$ at the collision is denoted by $c=c_{g}$ in anticipation of the connection with the concept of group velocity.

The conditions (4.2) tell us that the algebraic multiplicity of $c_{g}$ is two. For Hermitian matrices the geometric multiplicity would also be two. However, Hermitian matrix pencils, in the indefinite case, can have non-trivial Jordan chains (Gohberg et al. 2005). This property also carries over to Hermitian quadratic matrix polynomials (Gohberg et al. 1980). Here, we are interested in the case where the geometric multiplicity of $\mathbf{E}\left(c_{g}\right)$ is one

$$
\operatorname{Ker}\left(\mathbf{E}\left(c_{g}\right)\right)=\operatorname{span}\{\zeta\}
$$

To establish a Jordan chain, first look at the condition $\Delta^{\prime}\left(c_{g}\right)=0$ in terms of the properties of $\mathbf{E}(c)$,

$$
\begin{aligned}
\Delta^{\prime}\left(c_{g}\right)= & \left.\frac{d}{d c} \operatorname{det}[\mathbf{E}(c)]\right|_{c=c_{g}} \\
& =\left.\operatorname{Tr}\left(\mathbf{E}(c)^{\#} \mathbf{E}^{\prime}(c)\right)\right|_{c=c_{g}},
\end{aligned}
$$

where $\mathbf{E}(c)^{\#}$ is the adjugate (Magnus and Neudecker 1988). Now use the fact that $\mathbf{E}\left(c_{g}\right)$ has rank one and the nonzero eigenvalue is $\operatorname{Tr}\left(\mathbf{E}\left(c_{g}\right)\right)$,

$$
\mathbf{E}\left(c_{g}\right)^{\#}=\frac{\operatorname{Tr}\left(\mathbf{E}\left(c_{g}\right)\right)}{\|\zeta\|^{2}} \zeta \zeta^{T}
$$

This formula can be verified by direct calculation (see also Magnus and Neudecker 1988). Then,

$$
\Delta^{\prime}\left(c_{g}\right)=\left.\operatorname{Tr}\left(\mathbf{E}(c)^{\#} \mathbf{E}^{\prime}(c)\right)\right|_{c=c_{g}}=\frac{\operatorname{Tr}\left(\mathbf{E}\left(c_{g}\right)\right)}{\|\zeta\|^{2}}\left\langle\zeta, \mathbf{E}^{\prime}\left(c_{g}\right) \zeta\right\rangle,
$$


and so with the assumption (4.3),

$$
\Delta^{\prime}\left(c_{g}\right)=0 \Longleftrightarrow\left\langle\zeta, \mathbf{E}^{\prime}\left(c_{g}\right) \zeta\right\rangle=0 .
$$

Now look at this condition from the viewpoint of solvability, as that is how it will arise in the nonlinear modulation theory. In the case of algebraic multiplicity two and geometric multiplicity one, a Jordan chain for a quadratic Hermitian matrix polynomial has the form

$$
\mathbf{E}\left(c_{g}\right) \zeta=0 \text { and } \mathbf{E}\left(c_{g}\right) \boldsymbol{\gamma}=-\mathbf{E}^{\prime}\left(c_{g}\right) \zeta
$$

for some $\boldsymbol{\gamma} \in \mathbb{R}^{N}$, if it exists (Gohberg et al. 1980). Since $\mathbf{E}\left(c_{g}\right)$ is Hermitian (in this case real and symmetric), the solvability condition is $\left\langle\zeta, \mathbf{E}^{\prime}\left(c_{g}\right) \zeta\right\rangle=0$ confirming (4.4). Writing out this condition,

$$
0=\left\langle\zeta, \mathbf{E}^{\prime}\left(c_{g}\right) \zeta\right\rangle=\left\langle\zeta,\left(2 c_{g} \mathrm{D}_{\omega} \mathbf{A}+\left(\mathrm{D}_{\omega} \mathbf{B}+\mathrm{D}_{\mathbf{k}} \mathbf{A}\right)\right) \zeta\right\rangle
$$

gives a defining equation for $c_{g}$

$$
c_{g}=-\frac{1}{2} \frac{\left\langle\zeta,\left(\mathrm{D}_{\mathbf{k}} \mathbf{A}+\mathrm{D}_{\omega} \mathbf{B}\right) \zeta\right\rangle}{\left\langle\zeta, \mathrm{D}_{\omega} \mathbf{A} \zeta\right\rangle} .
$$

Noting that $\mathrm{D}_{\omega} \mathbf{B}=\left(\mathrm{D}_{\mathbf{k}} \mathbf{A}\right)^{T}$, this formula simplifies to

$$
c_{g}=-\frac{\left\langle\zeta, \mathrm{D}_{\mathbf{k}} \mathbf{A} \zeta\right\rangle}{\left\langle\zeta, \mathrm{D}_{\omega} \mathbf{A} \zeta\right\rangle} .
$$

The notation $c_{g}$ is used as the derivative with respect to $\mathbf{k}$ over a derivative with respect to $\omega$ is reminiscent of the classical definition of group velocity.

Termination of the chain (4.5) at length two is assured if the following equation

$$
\mathbf{E}\left(c_{g}\right) \Upsilon=-\mathbf{E}^{\prime}\left(c_{g}\right) \boldsymbol{\gamma}-\frac{1}{2} \mathbf{E}^{\prime \prime}\left(c_{g}\right) \zeta
$$

is not solvable; that is

$$
\mu:=\left\langle\zeta, \mathbf{E}^{\prime}\left(c_{g}\right) \boldsymbol{\gamma}+\frac{1}{2} \mathbf{E}^{\prime \prime}\left(c_{g}\right) \zeta\right\rangle=\frac{1}{2}\left\langle\zeta, \mathbf{E}^{\prime \prime}\left(c_{g}\right) \zeta\right\rangle-\left\langle\boldsymbol{\gamma}, \mathbf{E}\left(c_{g}\right) \boldsymbol{\gamma}\right\rangle \neq 0
$$

where (4.5) has been used. This expression is called $\mu$ as another remarkable result in the nonlinear theory is that this coefficient is precisely the $\mu$ that appears as the coefficient of $U_{T T}$ in the emergent two-way Boussinesq equation (1.27). This connection will emerge in the nonlinear modulation theory.

Further properties of this Jordan chain, and the Jordan chains associated with the linear operator $\mathbf{L}$ are discussed in more detail in Sect. 6, after the nonlinear modulation theory is introduced. 


\section{Nonlinear Modulation at Coalescence}

For the nonlinear modulation near coalescing characteristics, the strategy is to introduce the ansatz (1.32), substitute into the Euler-Lagrange equation (2.3), expand everything in powers of $\varepsilon$, and set terms proportional to each order in $\varepsilon$ to zero. The key step here is identifying the form of the ansatz. The role of frame speed is inspired by the one-phase case in Bridges and Ratliff (2017), and the role of additional phase functions $\boldsymbol{\psi}$ and $\delta$ is inspired by Ratliff (2018a). These are included in the ansatz because they eliminate the need for functions that would appear from homogeneous solutions at each order. The proposed phase modulation is

$$
\Phi=\phi+\varepsilon \psi+\varepsilon^{2} \delta .
$$

Then, with

$$
\boldsymbol{\Omega}:=\boldsymbol{\phi}_{T} \quad \text { and } \quad \mathbf{q}:=\boldsymbol{\phi}_{X} \quad \Rightarrow \quad \mathbf{q}_{T}-\boldsymbol{\Omega}_{X}=0,
$$

and

$$
X=\varepsilon\left(x+c_{g} t\right), \quad T=\varepsilon^{2} t,
$$

the complete proposed ansatz (1.32) is

$$
\begin{gathered}
Z(x, t)=\widehat{Z}\left(\boldsymbol{\theta}+\varepsilon \boldsymbol{\phi}+\varepsilon^{2} \boldsymbol{\psi}+\varepsilon^{3} \boldsymbol{\delta}, \mathbf{k}+\varepsilon^{2} \mathbf{q}+\varepsilon^{3} \boldsymbol{\psi}_{X}+\varepsilon^{4} \boldsymbol{\delta}_{X},\right. \\
\left.\boldsymbol{\omega}+\varepsilon^{2} c_{g} \mathbf{q}+\varepsilon^{3}\left(\boldsymbol{\Omega}+c_{g} \boldsymbol{\psi}_{X}\right)+\varepsilon^{4}\left(\boldsymbol{\psi}_{T}+c_{g} \boldsymbol{\delta}_{X}\right)+\varepsilon^{5} \boldsymbol{\delta}_{T}\right) \\
+\varepsilon^{3} W(\boldsymbol{\theta}, X, T ; \varepsilon) .
\end{gathered}
$$

where $\boldsymbol{\theta}, \boldsymbol{\phi}, \boldsymbol{\psi}, \boldsymbol{\delta}, \mathbf{q}$, and $\boldsymbol{\Omega}$ are all functions of $X$ and $T$ defined in (5.3), and $c_{g}$ is defined in (4.7). For ease, expand $W$ in an asymptotic series,

$$
W(\boldsymbol{\theta}, X, T, \varepsilon)=W_{3}(\boldsymbol{\theta}, X, T)+\varepsilon W_{4}(\boldsymbol{\theta}, X, T)+\varepsilon^{2} W_{5}(\boldsymbol{\theta}, X, T)+\cdots .
$$

The remainder $W$ could be defined as $W\left(\boldsymbol{\theta}+\varepsilon \boldsymbol{\phi}+\varepsilon^{2} \boldsymbol{\psi}+\varepsilon^{3} \boldsymbol{\delta}, X, T, \varepsilon\right)$, to synchronize with the form of the modulation of the basic state, but is equivalent to the above formulation: expansion of $W$ in a Taylor series in $\varepsilon$ just changes the form of $W_{j}$ at each order, but the overall expansion gives equivalent results.

Although the ansatz (5.4) is new, the expansion and substitution strategy is similar to our previous papers on multiphase modulation (Ratliff and Bridges 2016; Ratliff 2017a, 2018a) and the single phase coalescing characteristics (Bridges and Ratliff 2017) and so only the key new points are highlighted. For example, at $\varepsilon^{0}$ order the governing equation for $\widehat{Z}$ in (2.7) is recovered. At $\varepsilon^{1}$ and $\varepsilon^{2}$ order the generic 2-term Jordan chain in (2.11) is recovered as in the preceding works.

At third order in $\varepsilon$, after simplification, the system is

$$
\mathbf{L} W_{3}=\sum_{j=1}^{N} \partial_{X} q_{j} \mathbf{K}\left(\widehat{Z}_{k_{j}}+c_{g} \widehat{Z}_{\omega_{j}}\right)
$$


where

$$
\mathbf{K}:=\mathbf{J}+c_{g} \mathbf{M},
$$

and $c_{g}$ is defined in (4.7). Applying the solvability condition (2.13)-(5.5) gives

$$
\sum_{j=1}^{N} \partial_{X} q_{j}\left\langle\widehat{Z}_{\theta_{i}},\left(\mathbf{J}+c_{g} \mathbf{M}\right)\left(\widehat{Z}_{k_{j}}+c_{g} \widehat{Z}_{\omega_{j}}\right)\right\rangle=0, \quad i=1, \ldots, N
$$

or, after using the conversions from the structure operators $\mathbf{J}, \mathbf{M}$ to the functionals $\mathscr{A}_{j}, \mathscr{B}_{j}$ in $(2.19 \mathrm{~b})-(2.19 \mathrm{c})$, the solvability condition can be written in the illuminating vector form

$$
\left[\mathrm{D}_{\mathbf{k}} \mathbf{B}+c_{g}\left(\mathrm{D}_{\omega} \mathbf{B}+\mathrm{D}_{\mathbf{k}} \mathbf{A}\right)+c_{g}^{2} \mathrm{D}_{\omega} \mathbf{A}\right] \mathbf{q}_{X}=\mathbf{0} .
$$

Hence, for solvability of (5.5) it is required that $\mathbf{q}_{X}$ is in the kernel of $\mathbf{E}\left(c_{g}\right)$,

$$
\mathbf{q}_{X}=U_{X} \zeta \Rightarrow \mathbf{q}=U(X, T) \zeta+\mathbf{a}(T)
$$

for some scalar-valued function $U(X, T)$. It can be confirmed a posteriori that $\mathbf{a}(T)$ does not contribute to the leading order result and can be neglected. Hence,

$$
\mathbf{q}=U(X, T) \zeta
$$

It is this scalar-valued function $U(X, T)$ that will ultimately be found to be governed by the two-way Boussinesq equation (1.27).

With the solvability condition satisfied, and the expression for $\mathbf{q}$ in (5.8), the complete solution at third order is

$$
W_{3}=U_{X} \mathbf{v}_{3} \text {, with } \mathbf{L v}_{3}=\mathbf{K v}_{2} \text {. }
$$

An arbitrary amount of homogeneous solution can be added to $W_{3}$ but it is already incorporated into the functions $\delta$ and $\boldsymbol{\psi}$ in the ansatz. The equation $\mathbf{L v}_{3}=\mathbf{K} \mathbf{v}_{2}$ foreshadows a Jordan chain theory. The beginnings of the chain are in (2.11) which can be re-written as $\mathbf{L} \mathbf{v}_{1}=0$ and $\mathbf{L} \mathbf{v}_{2}=\mathbf{K} \mathbf{v}_{1}$. This Jordan chain theory is developed in Sect. 6. 


\subsection{Fourth Order}

After simplification, the equation at fourth order is

$$
\begin{array}{r}
\mathbf{L}\left(W_{4}-U_{X} \sum_{i=1}^{N} \phi_{i}\left(\mathbf{v}_{3}\right)_{\theta_{i}}\right)=U_{X X} \mathbf{K} \mathbf{v}_{3}+\sum_{j=1}^{N}\left(\psi_{j}\right)_{X X} \mathbf{K}\left(\widehat{Z}_{k_{j}}+c_{g} \widehat{Z}_{\omega_{j}}\right) \\
+U_{T} \sum_{j=1}^{N} \zeta_{j}\left(\mathbf{J} \widehat{Z}_{\omega_{j}}+\mathbf{M} \widehat{Z}_{k_{j}}+2 c_{g} \mathbf{M} \widehat{Z}_{\omega_{j}}\right) .
\end{array}
$$

The first inhomogeneous term feeds into the Jordan chain argument as it is of the form $\mathbf{L} \mathbf{v}_{4}=\mathbf{K} \mathbf{v}_{3}$, for some $\mathbf{v}_{4}$. For the other two inhomogeneous terms, apply the solvability conditions (2.13), and use the identities (2.19b)-(2.19c), to obtain

$$
\mathbf{E}\left(c_{g}\right) \psi_{X X}+\underbrace{\left[\left(\mathrm{D}_{\mathbf{k}} \mathbf{A}+\mathrm{D}_{\omega} \mathbf{B}\right)+2 c_{g} \mathrm{D}_{\omega} \mathbf{A}\right]}_{\mathbf{E}^{\prime}\left(c_{g}\right)} \zeta U_{T}=\mathbf{0} .
$$

This equation is of the form (4.5); that is, the Jordan chain associated with $\mathbf{E}(c)$. The theory of this Jordan chain is developed in Sect. 6.2. Here, it is sufficient to use the argument presented in (4.5) and (4.7) for the chain $(\boldsymbol{\zeta}, \boldsymbol{\gamma})$ of $\mathbf{E}\left(c_{g}\right)$. Applying that theory gives

$$
\boldsymbol{\psi}_{X X}=\boldsymbol{\gamma} U_{T}\left(\bmod \operatorname{Ker}\left(\mathbf{E}\left(c_{g}\right)\right)\right)
$$

where "mod" signifies that an arbitrary amount of homogeneous solution can be included. This homogeneous solution can be neglected as it does not enter at fifth order. Thus the solution at fourth order is

$$
W_{4}=U_{X X} \mathbf{v}_{4}+U_{T} \Xi+U_{X} \sum_{j=1}^{N} \phi_{j}\left(\xi_{5}\right)_{\theta_{j}} \quad(\bmod \operatorname{Ker}(\mathbf{L})),
$$

with

$$
\mathbf{L} \Xi=\sum_{j=1}^{N}\left[\zeta_{j}\left(\widehat{J}_{\omega_{j}}+\mathbf{M} \widehat{Z}_{k_{j}}+2 c_{g} \mathbf{M} \widehat{Z}_{\omega_{j}}\right)+\gamma_{j} \mathbf{K}\left(\widehat{Z}_{k_{j}}+c_{g} \widehat{Z}_{\omega_{j}}\right)\right]
$$

Fortunately, this equation does not need to be solved explicitly. It feeds into the fifth-order solution, and ultimately generates formulae for the coefficients, but these formulae will be obtained without an explicit expression for $\Xi$. 


\subsection{Fifth Order}

At fifth order, after combining terms and simplifying, the equations are

$$
\begin{aligned}
\mathbf{L} \widetilde{W}_{5}= & U_{X X X} \mathbf{K} \mathbf{v}_{4}+\sum_{i=1}^{N}\left[\left(\Omega_{i}\right)_{T} \mathbf{M} \widehat{Z}_{\omega_{i}}+\left(\delta_{i}\right)_{X} \mathbf{K}\left(\widehat{Z}_{k_{i}}+c_{g} \widehat{Z}_{\omega_{i}}\right)\right] \\
& +U_{X T}\left(\mathbf{J} \Xi+\mathbf{M} \mathbf{v}_{3}\right)+\sum_{i=1}^{N}\left(\psi_{i}\right)_{X T}\left(\mathbf{J} \widehat{Z}_{\omega_{i}}+\mathbf{M} \widehat{Z}_{k_{i}}+2 c_{g} \mathbf{M} \widehat{Z}_{\omega_{i}}\right) \\
& +U U_{X} \sum_{i=1}^{N}\left[\mathbf{K}\left(\mathbf{v}_{3}\right)_{\theta_{i}}-\mathrm{D}^{3} S(\widehat{Z})\left(\mathbf{v}_{3}, \widehat{Z}_{k_{i}}+c_{g} \widehat{Z}_{\omega_{i}}\right)\right. \\
& \left.+\sum_{j=1}^{N} \mathbf{K}\left(\widehat{Z}_{k_{i} k_{j}}+c_{g} \widehat{Z}_{\omega_{i} k_{j}}+c_{g} \widehat{Z}_{k_{i} \omega_{j}}+c_{g}^{2} \widehat{Z}_{\omega_{i} \omega_{j}}\right)\right]
\end{aligned}
$$

The tilde above $W_{5}$ term indicates that the preimage of all terms lying in the range of $\mathbf{L}$ from the right-hand side have been absorbed (e.g. terms that would vanish identically under the solvability conditions). These terms come into play only at higher order.

It is the solvability condition for this fifth-order equation that will deliver the modulation equation for $U(X, T)$. However, solvability is a multistage process. There are $N$ solvability conditions associated with the operator $\mathbf{L}$, leading to a vector-valued equation. A secondary solvability condition, associated with the operator $\mathbf{E}\left(c_{g}\right)$, will reduce vector equation to the scalar two-way Boussinesq equation.

First establish the vector solvability condition. Apply the L-solvability (2.13) condition to the right-hand side of (5.15) term by term. Solvability of the $U_{X X X}$ term generates the vector

$$
\left(\begin{array}{c}
\left\langle\left\langle\widehat{Z}_{\theta_{1}}, \mathbf{K} \mathbf{v}_{4}\right\rangle\right\rangle \\
\vdots \\
\left\langle\left\langle\widehat{Z}_{\theta_{N}}, \mathbf{K} \mathbf{v}_{4}\right\rangle\right.
\end{array}\right) U_{X X X}:=-\mathbf{T} U_{X X X}
$$

We will see that this vector is nonzero since, by hypothesis, the Jordan chain $\left(\mathbf{v}_{1}, \ldots, \mathbf{v}_{4}\right)$ has length four. This is discussed in Sect. 6. Solvability of the $\left(\Omega_{i}\right)_{T}$ terms leads to the matrix term

$$
\left(\begin{array}{ccc}
\left\langle\left\langle\widehat{Z}_{\theta_{1}}, \mathbf{M} \widehat{Z}_{\omega_{1}}\right\rangle\right\rangle & \cdots & \left\langle\left\langle\widehat{Z}_{\theta_{1}}, \mathbf{M} \widehat{Z}_{\omega_{N}}\right\rangle\right. \\
\vdots & \ddots & \vdots \\
\left.\left\langle\widehat{Z}_{\theta_{N}}, \mathbf{M} \widehat{Z}_{\omega_{1}}\right\rangle\right\rangle & \cdots & \left\langle\left\langle\widehat{Z}_{\theta_{N}}, \mathbf{M} \widehat{Z}_{\omega_{N}}\right\rangle\right.
\end{array}\right) \boldsymbol{\Omega}_{T} \equiv-\mathrm{D}_{\boldsymbol{\omega}} \mathbf{A} \boldsymbol{\Omega}_{T} .
$$

The terms containing $\delta_{i}$ give

$$
\left(\begin{array}{ccc}
\left\langle\left\langle\widehat{Z}_{\theta_{1}}, \mathbf{K}\left(\widehat{Z}_{k_{1}}+c_{g} \widehat{Z}_{\omega_{1}}\right)\right\rangle\right\rangle & \cdots & \left\langle\left\langle\widehat{Z}_{\theta_{1}}, \mathbf{K}\left(\widehat{Z}_{k_{N}}+c_{g} \widehat{Z}_{\omega_{N}}\right)\right\rangle\right\rangle \\
\vdots & \ddots & \vdots \\
\left.\left\langle\widehat{Z}_{\theta_{N}}, \mathbf{K}\left(\widehat{Z}_{k_{1}}+c_{g} \widehat{Z}_{\omega_{1}}\right)\right\rangle\right\rangle & \cdots & \left\langle\left\langle\widehat{Z}_{\theta_{N}}, \mathbf{K}\left(\widehat{Z}_{k_{N}}+c_{g} \widehat{Z}_{\omega_{N}}\right)\right\rangle\right\rangle
\end{array}\right) \boldsymbol{\delta}_{X X}=-\mathbf{E}\left(c_{g}\right) \boldsymbol{\delta}_{X X} .
$$


The terms involving $\left(\psi_{i}\right)_{X T}$ are similar to those seen at fourth order, and generate

$$
\begin{array}{r}
\left(\begin{array}{ccc}
\left.\left\langle\widehat{Z}_{\theta_{1}}, \mathbf{J} \widehat{Z}_{\omega_{1}}+\mathbf{M} \widehat{Z}_{k_{1}}+2 c_{g} \mathbf{M} \widehat{Z}_{\omega_{1}}\right\rangle\right\rangle & \cdots & \left\langle\left\langle\widehat{Z}_{\theta_{1}}, \mathbf{J} \widehat{Z}_{\omega_{N}}+\mathbf{M} \widehat{Z}_{k_{N}}+2 c_{g} \mathbf{M} \widehat{Z}_{\omega_{N}}\right\rangle\right. \\
\vdots & \ddots & \vdots \\
\left\langle\widehat{Z}_{\theta_{N}}, \mathbf{J} \widehat{Z}_{\omega_{1}}+\mathbf{M} \widehat{Z}_{k_{1}}+2 c_{g} \mathbf{M} \widehat{Z}_{\omega_{1}}\right\rangle & \cdots & \left\langle\left\langle\widehat{Z}_{\theta_{N}}, \mathbf{J} \widehat{Z}_{\omega_{N}}+\mathbf{M} \widehat{Z}_{k_{N}}+2 c_{g} \mathbf{M} \widehat{Z}_{\omega_{N}}\right\rangle\right.
\end{array}\right) \boldsymbol{\psi}_{X T} \\
=-\left[\left(\mathrm{D}_{\mathbf{k}} \mathbf{A}+\mathrm{D}_{\omega} \mathbf{B}\right)+2 c_{g} \mathrm{D}_{\omega} \mathbf{A}\right] \boldsymbol{\psi}_{X T} \\
=-\mathbf{E}^{\prime}\left(c_{g}\right) \boldsymbol{\psi}_{X T} .
\end{array}
$$

The coefficient of the nonlinear term $U U_{X}$ simplifies to

$$
\begin{array}{r}
-\left(\mathrm{D}_{\mathbf{k}}^{2} \mathbf{B}+c_{g}\left(2 \mathrm{D}_{\mathbf{k}} \mathrm{D}_{\omega} \mathbf{B}+\mathrm{D}_{\mathbf{k}}^{2} \mathbf{A}\right)+c_{g}^{2}\left(2 \mathrm{D}_{\mathbf{k}} \mathrm{D}_{\omega} \mathbf{A}+\mathrm{D}_{\omega}^{2} \mathbf{B}\right)+c_{g}^{3} \mathrm{D}_{\omega}^{2} \mathbf{A}\right)(\zeta, \zeta) U U_{X} \\
:=-\mathbf{H}(\zeta, \zeta) U U_{X} .
\end{array}
$$

When $c_{g}=0$ the vector function $\mathbf{H}(\zeta, \zeta)$ reduces to $\mathbf{H}(\zeta, \zeta)=\mathrm{D}_{\mathbf{k}}^{2} \mathbf{B}(\zeta, \zeta)$ which is the form found in reduction of multiphase modulation to the KdV equation in Ratliff and Bridges (2016), Ratliff (2018b).

Collecting these terms gives the vector form of the solvability condition for (5.15)

$$
\mathbf{E}\left(c_{g}\right) \boldsymbol{\delta}_{X X}+\mathrm{D}_{\omega} \mathbf{A} \Omega_{T}+\mathbf{E}^{\prime}\left(c_{g}\right) \boldsymbol{\psi}_{X T}+\mathbf{T} U_{X X X}+\mathbf{H}(\zeta, \zeta) U U_{X}=\mathbf{0} .
$$

This equation is interesting in itself, but it is not closed due to the presence of the $\boldsymbol{\delta}_{X X}$ term and the $\psi_{X T}$ term. However, the $\delta_{X X}$ term is acted on by $\mathbf{E}\left(c_{g}\right)$ and so this term vanishes when the equation is projected onto the kernel of $\mathbf{E}\left(c_{g}\right)$. Therefore, split $\mathbb{R}^{N}$ as

$$
\mathbb{R}^{N}=\operatorname{span}\{\zeta\} \oplus \mathbb{R}^{N-1}
$$

The projection of (5.21) onto the complement of $\operatorname{Ker}\left(\mathbf{E}\left(c_{g}\right)\right)$ still contains the $\boldsymbol{\delta}_{X X}$ term but this part carries over to higher order.

With this splitting in mind, act on (5.21) with $\zeta^{T}$,

$$
\zeta^{T} \mathbf{E}\left(c_{g}\right) \boldsymbol{\delta}_{X X}+\zeta^{T} \mathrm{D}_{\omega} \mathbf{A} \boldsymbol{\Omega}_{T}+\zeta^{T} \mathbf{E}^{\prime}\left(c_{g}\right) \boldsymbol{\psi}_{X T}+\zeta^{T} \mathbf{T} U_{X X X}+\zeta^{T} \mathbf{H}(\zeta, \zeta) U U_{X}=0 .
$$

Defining

$$
\kappa=\zeta^{T} \mathbf{H}(\zeta, \zeta) \text { and } \mathscr{K}=\zeta^{T} \mathbf{T}=\left\langle\left\langle\mathbf{K v}_{1}, \mathbf{v}_{4}\right\rangle\right.
$$

and noting that the coefficient of the $\boldsymbol{\delta}_{X X}$ term now vanishes as $\mathbf{E}\left(c_{g}\right)$ is symmetric, (5.22) simplifies the vector equation to

$$
\zeta^{T} \mathrm{D}_{\omega} \mathbf{A} \boldsymbol{\Omega}_{T}+\zeta^{T} \mathbf{E}^{\prime}\left(c_{g}\right) \boldsymbol{\psi}_{X T}+\kappa U U_{X}+\mathscr{K} U_{X X X}=0
$$


This equation is closed by first differentiating with respect to $X$,

$$
\zeta^{T} \mathrm{D}_{\omega} \mathbf{A} \boldsymbol{\Omega}_{X T}+\zeta^{T} \mathbf{E}^{\prime}\left(c_{g}\right) \boldsymbol{\psi}_{X X T}+\kappa\left(U U_{X}\right)_{X}+\mathscr{K} U_{X X X X}=0,
$$

and applying conservation of waves and the $\psi-U$ equation (5.12),

$$
\boldsymbol{\Omega}_{X T}=\mathbf{q}_{T T}=\zeta U_{T T} \text { and } \boldsymbol{\psi}_{X X T}=\boldsymbol{\gamma} U_{T T} .
$$

Hence, the final form of the two-way Boussinesq equation is

$$
\mu U_{T T}+\kappa\left(U U_{X}\right)_{X}+\mathscr{K} U_{X X X X}=0,
$$

with

$$
\mu=\zeta^{T} \mathrm{D}_{\omega} \mathbf{A} \zeta+\zeta^{T}\left[\left(\mathrm{D}_{\mathbf{k}} \mathbf{A}+\mathrm{D}_{\omega} \mathbf{B}-2 c_{g} \mathrm{D}_{\omega} \mathbf{A}\right] \boldsymbol{\gamma}\right.
$$

Another way to write this is to use $\mathbf{E}\left(c_{g}\right)$,

$$
\mu=\frac{1}{2} \zeta^{T} \mathbf{E}^{\prime \prime}\left(c_{g}\right) \zeta+\zeta^{T} \mathbf{E}^{\prime}\left(c_{g}\right) \boldsymbol{\gamma}
$$

emphasizing that $\mu \neq 0$ is the termination condition for the $(\boldsymbol{\zeta}, \boldsymbol{\gamma})$ Jordan chain.

Comparing $\zeta^{T} \mathbf{H}(\zeta, \zeta)$ with (2.24) shows that

$$
\kappa=\zeta^{T} \mathbf{H}(\zeta, \zeta)=\left.\frac{d^{3}}{d s^{3}} \mathscr{L}\left(\boldsymbol{\omega}+s c_{g} \boldsymbol{\zeta}, \mathbf{k}+s \zeta\right)\right|_{s=0} .
$$

The emergent two-way Boussinesq equation is non-degenerate when $\mu, \kappa$ and $\mathscr{K}$ are nonzero. The coefficient $\mu$ is nonzero when the Jordan chain for $\mathbf{E}\left(c_{g}\right)$ in (4.5) terminates at two. The coefficient $\kappa$ is assumed to be nonzero. If it is zero, then it is expected that re-modulation will lead to a cubic nonlinearity (El et al. 2017; Ratliff and Bridges 2018; Ratliff 2018b). The coefficient of dispersion is nonzero if the Jordan chain in (6.11) terminates at four. If $\mathscr{K}$ vanishes, then a longer Jordan chain will emerge. Re-modulation in this case is expected to lead to higher-order dispersive terms emerging (e.g. sixth-order dispersion, as in Sprenger and Hoefer 2017; Ratliff 2017b).

The above result does not provide any information about convergence of the ansatz (5.4) as a Taylor series in $\varepsilon$. However, the asymptotic validity of this ansatz is confirmed by the above results; that is, the ansatz (5.4) satisfies the governing equations exactly up to $\mathcal{O}\left(\varepsilon^{5}\right)$,

$$
\left\|\mathbf{M} Z_{t}+\mathbf{J} Z_{x}=\nabla S(Z)\right\|=\mathcal{O}\left(\varepsilon^{6}\right) \text { as } \varepsilon \rightarrow 0 .
$$

For generic multiphase WMT, a rigorous proof of validity has been given for CNLS (Bridges et al. 2020), but a rigorous proof of validity in the case of coalescing characteristics is an open problem. 
To summarize, the starting point is a PDE generated by a Lagrangian with a multiphase basic state. It is assumed that, at some parameter value, a pair of coalescing characteristics arises in the linearized Whitham equations. These coalescing characteristics generate several Jordan chains. A modulation ansatz of the form (5.4) then leads to a scalar two-way Boussinesq equation (5.24) with coefficients $\mu, \kappa$, and $\mathscr{K}$ all determined from abstract properties of the averaged Lagrangian. The fundamental idea is that the original PDE is reduced to a simpler PDE that can be analysed in some detail. Some of the solutions of this reduced two-way Boussinesq equation are anticipated in Sect. 7.

\section{Coalescing Characteristics and Jordan Chains}

Jordan chains play an important part throughout the steps of the derivation of the nonlinear modulation equations. In this section, some of the properties of these Jordan chains are examined in more detail.

There are two key linear operators: $\mathbf{L}$ and $\mathbf{E}(c)$. The operator $\mathbf{L}$, associated with the linearization of the Euler-Lagrange equation (2.3), generates a Jordan chain theory that starts with

$$
\mathbf{L} \xi_{j}=0 \quad \text { with } \quad \xi_{j}:=\frac{\partial \widehat{Z}}{\partial \theta_{j}}, j=1, \ldots, N
$$

and

$$
\begin{array}{cc}
\mathbf{L} \xi_{1}=0, \mathbf{L} \xi_{N+1} & =\mathbf{J} \xi_{1} \\
\vdots & \vdots \\
\mathbf{L} \xi_{N}=0, \mathbf{L} \xi_{2 N} & =\mathbf{J} \xi_{N},
\end{array}
$$

which follow from (2.11) with

$$
\xi_{N+j}:=\frac{\partial \widehat{Z}}{\partial k_{j}}, \quad j=1, \ldots, N .
$$

With the assumption that $\operatorname{Ker}(\mathbf{L})=\operatorname{span}\left\{\xi_{1}, \ldots, \xi_{N}\right\}$, there are $N$ Jordan blocks each of dimension two.

The operator $\mathbf{E}\left(c_{g}\right)$ generates another Jordan chain which can be discussed independently of the $\mathbf{L}$-chains, but feeds into solvability of the $\mathbf{L}$ chains, and it starts with

$$
\mathbf{E}(c) \zeta=0 \text {. }
$$

Generically, E(c) has a single Jordan block of dimension one. At isolated values, for example at $c=c_{g}$, the Jordan block increases to dimension two. Even though $\mathbf{E}(c)$ is Hermitian for any real $c$, it still generates a non-trivial Jordan chain due to the fact that $c$ appears nonlinearly. 
The theory needed to extend these two Jordan chains is well established in the literature. The above $\mathbf{L}$-chains are $\mathbf{J}$-symplectic Jordan chains and this theory goes back to Williamson (1936), and the theory of Jordan chains for quadratic Hermitian matrix pencils is developed in Gohberg et al. (1980).

However, things get complicated when we realize that the linear operator $\mathbf{L}$ has both J-Jordan chains and M-Jordan chains. From (2.11), it follows that there exist M-Jordan chains of the form

$$
\begin{array}{cc}
\mathbf{L} \xi_{1}=0, \mathbf{L} \eta_{N+1}=\mathbf{M} \xi_{1} \\
\vdots & \vdots \\
\mathbf{L} \xi_{N}=0, \mathbf{L} \eta_{2 N} & =\mathbf{M} \xi_{N}
\end{array}
$$

which follow from (2.11) with

$$
\eta_{N+j}:=\frac{\partial \widehat{Z}}{\partial \omega_{j}}, \quad j=1, \ldots, N
$$

The J-chains (6.2) have length greater than two if at least one of the following

$$
\mathbf{L} \chi_{j}=\mathbf{J} \xi_{N+j}, \quad j=1, \ldots, N
$$

is solvable, and termination at two is associated with non-solvability of all $N$ of these equations. Similarly, the M-chains have length greater than two if at least one of the following

$$
\mathbf{L} \chi_{j}=\mathbf{M} \eta_{N+j}, \quad j=1, \ldots, N
$$

is solvable, and termination at two is associated with non-solvability. The $N$ chains in (6.2) and (6.4) can also be mixed, by taking the first elements to be linear combinations of $\xi_{1}, \ldots, \xi_{N}$, and this turns out to be useful for the modulation theory.

Combining all the possibilities for both $\mathbf{J}$-chains and $\mathbf{M}$-chains, the most general extension of the Jordan chains is that there exists a vector $\Xi$ satisfying

$$
\mathbf{L} \Xi=\sum_{j=1}^{N}\left(a_{j} \mathbf{M} \eta_{N+j}+b_{j} \mathbf{M} \xi_{N+j}+c_{j} \mathbf{J} \eta_{N+j}+d_{j} \mathbf{J} \xi_{N+j}\right)
$$

No theory exists for Jordan chains of this type. The closest approximation is the Jordan chain theory for multiparameter eigenvalue problems (e.g. Binding and Volkmer 1996 and its citation trail), but that does not apply here either. We will be able to develop a satisfactory theory for multi-dimensional Jordan chains of this type to cover the cases needed in the modulation theory, but a complete and general theory for multidimensional Jordan chains of this type is outside the scope of this paper. 
A solution $\Xi$ of (6.5) exists if this equation is solvable, and it is solvable if and only if the $4 N$ constants $a_{j}, b_{j}, c_{j}, d_{j}$ for $j=1, \ldots, N$, satisfy

$$
\left\langle\xi_{\ell}, \sum_{j=1}^{N}\left(a_{j} \mathbf{M} \eta_{N+j}+b_{j} \mathbf{M} \xi_{N+j}+c_{j} \mathbf{J} \eta_{N+j}+d_{j} \mathbf{J} \xi_{N+j}\right)\right\rangle=0, \quad \ell=1, \ldots, N
$$

These equations can be simplified by using the identities in Sect. 2.1.2, giving

$$
\left[\mathrm{D}_{\omega} \mathbf{A}\right] \mathbf{a}+\left[\mathrm{D}_{\mathbf{k}} \mathbf{A}\right] \mathbf{b}+\left[\mathrm{D}_{\omega} \mathbf{B}\right] \mathbf{c}+\left[\mathrm{D}_{\mathbf{k}} \mathbf{B}\right] \mathbf{d}=\mathbf{0}
$$

where

$$
\mathbf{a}:=\left(\begin{array}{c}
a_{1} \\
\vdots \\
a_{N}
\end{array}\right), \quad \mathbf{b}:=\left(\begin{array}{c}
b_{1} \\
\vdots \\
b_{N}
\end{array}\right), \quad \mathbf{c}:=\left(\begin{array}{c}
c_{1} \\
\vdots \\
c_{N}
\end{array}\right), \quad \mathbf{d}:=\left(\begin{array}{c}
d_{1} \\
\vdots \\
d_{N}
\end{array}\right)
$$

Hence, if there exists values of these $4 N$ constants for which Eq. (6.6) has a non-trivial solution, then $\Xi$ is the next vector in the generalized Jordan chain.

A general theory considering all possible Jordan chains emanating from the condition (6.6) is outside the scope of this paper. However, we will highlight special cases that appear in the nonlinear modulation theory. The case $\mathbf{a}=\mathbf{c}=0$ (a pure $\mathbf{J}$-chain) appears in the nonlinear modulation theory associated with zero characteristics (Ratliff and Bridges 2016; Ratliff 2017a, 2018a), and the case $\mathbf{b}=\mathbf{d}=0$ is mathematically equivalent, and generates a pure $\mathbf{M}$-chain. Here, two new cases which intertwine the $\mathbf{J}$ and $\mathbf{M}$ chains, and are required for the nonlinear modulation theory in this paper, will be highlighted.

\subsection{The Key Jordan Chain of Length Four}

Taking

$$
\mathbf{a}=c^{2} \zeta, \quad \mathbf{b}=\mathbf{c}=c \zeta \text { and } \mathbf{d}=\zeta
$$

reduces the solvability condition (6.6) to

$$
\left[c^{2} \mathrm{D}_{\omega} \mathbf{A}+c\left(\mathrm{D}_{\mathbf{k}} \mathbf{A}+\mathrm{D}_{\omega} \mathbf{B}\right)+\mathrm{D}_{\mathbf{k}} \mathbf{B}\right] \zeta=\mathbf{0} .
$$

Remarkably, this is precisely the equation for characteristics. In this case, the Jordan chain associated with $\mathbf{L}$ can continue when $\Delta(c)=0$ and $\zeta \in \operatorname{Ker}[\mathbf{E}(c)]$, the familiar condition (1.23) for the existence of a characteristic $c$. However, this construction does not imply that $c=c_{g}$, that equivalence will follow from another Jordan chain and it is considered in Sect. 6.2.

In the case (6.7) with (6.8), the Jordan chain intertwines the symplectic $\mathbf{J}$-chain and the symplectic M-chain. They can be combined to a new symplectic Jordan chain, 
based on the combined symplectic operator $\mathbf{J}+c \mathbf{M}$ and ultimately leads to the central Jordan chain that shows up in the nonlinear modulation theory.

Suppose first that $c$ is arbitrary, and see that the condition $c=c_{g}$ will arise as a condition to extend the Jordan chain in Sect. 6.2. For arbitrary $c$, there is still a geometric eigenvector $\zeta$ satisfying $\mathbf{E}(c) \zeta=0$. Express it in components, $\zeta=\left(\zeta_{1}, \ldots, \zeta_{N}\right)$, and re-number the generalized eigenvectors as follows,

$$
\begin{aligned}
\mathbf{v}_{1} & =\sum_{j=1}^{N} \zeta_{j} \partial \widehat{Z} / \partial \theta_{j} \\
\mathbf{v}_{2} & =\sum_{j=1}^{N} \zeta_{j}\left(\partial \widehat{Z} / \partial k_{j}+c \partial \widehat{Z} / \partial \omega_{j}\right),
\end{aligned}
$$

These two vectors satisfy

$$
\mathbf{L} \mathbf{v}_{1}=0 \quad \text { and } \quad \mathbf{L v}_{2}=(\mathbf{J}+c \mathbf{M}) \mathbf{v}_{1}
$$

This Jordan chain of length continues to length three if the following equation is solvable,

$$
\mathbf{L v}_{3}=(\mathbf{J}+c \mathbf{M}) \mathbf{v}_{2} .
$$

But, the existence of the $\mathbf{v}_{3}$ term is just a reformulation of the solvability condition (4.6) in terms of the new coordinates. To see this, write out the solvability condition for $\mathbf{v}_{3}$

$$
\left\langle\left\langle\widehat{Z}_{\theta_{j}},(\mathbf{J}+c \mathbf{M}) \mathbf{v}_{2}\right\rangle\right\rangle=0, \quad j=1, \ldots, N
$$

Using (6.9), and noting that

$$
\begin{aligned}
\left\langle\left\langle\widehat{Z}_{\theta_{j}},(\mathbf{J}+c \mathbf{M}) \mathbf{v}_{2}\right\rangle\right\rangle & =-\left\langle\left\langle(\mathbf{J}+c \mathbf{M}) \widehat{Z}_{\theta_{j}}, \mathbf{v}_{2}\right\rangle\right\rangle \\
& =-\left\langle\left\langle\mathbf{L}\left(\partial_{k_{j}} \widehat{Z}+c \partial_{\omega_{j}} \widehat{Z}\right), \mathbf{v}_{2}\right\rangle\right\rangle \\
& =-\left\langle\left\langle\left(\partial_{k_{j}} \widehat{Z}+c \partial_{\omega_{j}} \widehat{Z}\right), \mathbf{L} \mathbf{v}_{2}\right\rangle\right\rangle \\
& =-\left\langle\left\langle\left(\partial_{k_{j}} \widehat{Z}+c \partial_{\omega_{j}} \widehat{Z}\right),(\mathbf{J}+c \mathbf{M}) \mathbf{v}_{1}\right\rangle\right\rangle, \quad j=1, \ldots, N .
\end{aligned}
$$

Substituting for $\mathbf{v}_{1}$ and using the identities (2.19a)-(2.19c), generates precisely Eq. (6.8). Indeed it was working backwards from (6.8) that suggested the definitions (6.9). Since $\mathbf{L}$ is symmetric and $\mathbf{J}+c \mathbf{M}$ is skew-symmetric every Jordan chain has even length, assuring the existence of $\mathbf{v}_{4}$,

$$
\mathbf{L v}_{4}=(\mathbf{J}+c \mathbf{M}) \mathbf{v}_{3}
$$

It is assumed that the this four chain terminates; that is, the system

$$
\mathbf{L v}_{5}=(\mathbf{J}+c \mathbf{M}) \mathbf{v}_{4}
$$


is not solvable. The four chain

$$
\mathbf{L} \mathbf{v}_{j}=(\mathbf{J}+c \mathbf{M}) \mathbf{v}_{j-1}, \quad j=1, \ldots, 4,
$$

with $\mathbf{v}_{0}=0$ is the Jordan chain that plays a key role in the nonlinear modulation theory. The non-solvability of (6.10) also arises in the nonlinear modulation theory. It ensures that the coefficient of dispersion, $\mathscr{K}$, is nonzero.

In addition there are $N-1$ Jordan blocks of length two, but explicit expressions for these blocks are not needed in the nonlinear modulation theory. It is however assumed that they are each of length exactly two.

\subsection{Another Mixed Jordan Chain Defining $c_{g}$}

There is yet another Jordan chain, associated with $\mathbf{L}$, that arises in the nonlinear modulation theory and the solvability condition for this chain defines $c_{g}$. It is a special case of the solvability condition (6.6) obtained by taking

$$
\mathbf{a}=c^{2} \boldsymbol{\gamma}+2 c \zeta, \quad \mathbf{b}=\mathbf{c}=c \boldsymbol{\gamma}+\zeta, \quad \mathbf{d}=\boldsymbol{\gamma}
$$

Substitution of (6.12) into (6.6) and rearranging gives

$$
\left[\mathrm{D}_{\omega} \mathbf{A} c^{2}+\left(\mathrm{D}_{\mathbf{k}} \mathbf{A}+\mathrm{D}_{\omega} \mathbf{B}\right) c+\mathrm{D}_{\mathbf{k}} \mathbf{B}\right] \boldsymbol{\gamma}+\left[2 c \mathrm{D}_{\omega} \mathbf{A}+\left(\mathrm{D}_{\mathbf{k}} \mathbf{A}+\mathrm{D}_{\omega} \mathbf{B}\right)\right] \zeta=0
$$

or

$$
\mathbf{E}(c) \boldsymbol{\gamma}+\mathbf{E}^{\prime}(c) \zeta=0
$$

This equation is solvable for a fixed value of $c$ only, and the solvability condition

$$
\left\langle\zeta, \mathbf{E}^{\prime}\left(c_{g}\right) \zeta\right\rangle=0
$$

agrees with the definition of $c_{g}$ in (4.5) and (4.7). When $c=c_{g}$, the vectors $(\boldsymbol{\zeta}, \boldsymbol{\gamma})$ form a Jordan chain for $\mathbf{E}\left(c_{g}\right)$ of length two.

Suppose the solvability condition (6.13) and (6.14) is satisfied, then substitution back into (6.5) gives that

$$
\mathbf{L} \Xi=\sum_{i=1}^{N}\left[\zeta_{i}\left(\mathbf{J} \widehat{Z}_{\omega_{i}}+\mathbf{M} \widehat{Z}_{k_{i}}+2 c_{g} \mathbf{M} \widehat{Z}_{\omega_{i}}\right)+\gamma_{i}\left(\mathbf{J}+c_{g} \mathbf{M}\right)\left(\widehat{Z}_{k_{i}}+c_{g} \widehat{Z}_{\omega_{i}}\right)\right]
$$

It is this equation that arose in the modulation theory at fourth order (5.14) and working backwards we see that it is a special case of (6.5) and moreover solvability, with the expressions (6.12), is precisely the condition for the Jordan chain (6.14) of $\mathbf{E}\left(c_{g}\right)$. 
Further still, we can define another special case, which results in the criterion for the termination of this chain. This is achieved by setting

$$
\mathbf{a}=c_{g}^{2} \Upsilon+2 c_{g} \boldsymbol{\gamma}+\zeta, \quad \mathbf{b}=\mathbf{c}=c_{g} \Upsilon+\boldsymbol{\gamma}, \quad \mathbf{d}=\Upsilon
$$

Utilizing this in (6.6) and simplifying results in the system

$$
\mathbf{E}\left(c_{g}\right) \Upsilon+\mathbf{E}^{\prime}\left(c_{g}\right) \boldsymbol{\gamma}+\frac{1}{2} \mathbf{E}^{\prime \prime}\left(c_{g}\right) \zeta=0,
$$

which is precisely (4.9). The assumption made here is that this chain is of length two, and so the right hand side of (4.9) does not lie in the range of $\mathbf{L}$. Thus, by appealing to solvability one recovers the condition that

$$
\mu:=\left\langle\zeta, \mathbf{E}^{\prime}\left(c_{g}\right) \boldsymbol{\gamma}+\frac{1}{2} \mathbf{E}^{\prime \prime}\left(c_{g}\right) \zeta\right\rangle=\frac{1}{2}\left\langle\zeta, \mathbf{E}^{\prime \prime}\left(c_{g}\right) \zeta\right\rangle-\left\langle\boldsymbol{\gamma}, \mathbf{E}\left(c_{g}\right) \boldsymbol{\gamma}\right\rangle \neq 0
$$

and therefore completing the connection between $\mu$ and the termination of this mixed Jordan chain. Within the modulation theory, this corresponds to the system

$\mathbf{L} \Gamma=\sum_{i=1}^{N}\left[\zeta_{i} \mathbf{M} \widehat{Z}_{\omega_{i}}+\gamma_{i}\left(\mathbf{J} \widehat{Z}_{\omega_{i}}+\mathbf{M} \widehat{Z}_{k_{i}}+2 c_{g} \mathbf{M} \widehat{Z}_{\omega_{i}}\right)+\Upsilon_{i}\left(\mathbf{J}+c_{g} \mathbf{M}\right)\left(\widehat{Z}_{k_{i}}+c_{g} \widehat{Z}_{\omega_{i}}\right)\right]$

being unsolvable for $\Gamma$, and what ultimately leads to the coefficient of the time derivative term in the emergent two-way Boussinesq equation.

We have only scratched the surface of the possible solvability conditions and attendant Jordan chains associated with (6.5). However, we have all the Jordan chains needed for the nonlinear modulation theory.

\section{Properties of the Two-way Boussinesq Equation}

Once the modulation equation is derived in a specific context, analysis of the solutions of the two-way Boussinesq equation (5.24) gives information about the nature of solutions in the nonlinear problem near coalescence.

The two-way Boussinesq equation is valid at $c=c_{g}$. At least one parameter needs to be varied to obtain the coalescence. That parameter can be a one-parameter path through the four-dimensional frequency-wavenumber $(\boldsymbol{\omega}, \mathbf{k})$ space, or it could be a perturbation of the frame speed $c=c_{g}+\mathcal{O}\left(\varepsilon^{2}\right)$. Unfolding the singularity generates a term of the form $v U_{X X}$ in (5.24), regardless of the precise perturbation path (this can be shown by perturbing the linearized generic Whitham equations). Therefore, the full modulation equation in the neighbourhood of the coalescence is

$$
\mu U_{T T}+v U_{X X}+\kappa\left(U U_{X}\right)_{X}+\mathscr{K} U_{X X X X}=0,
$$

where $v$ is an order one constant. 

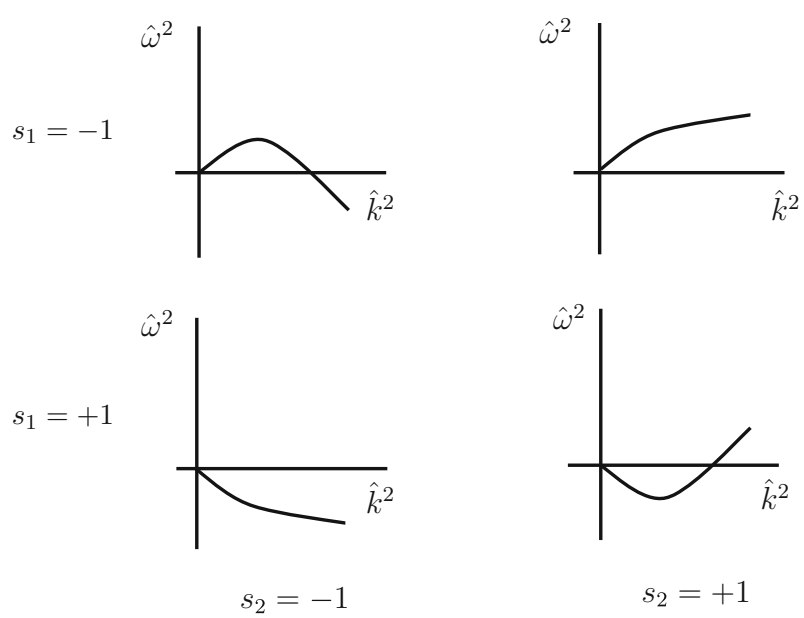

Fig. 2 The four qualitative cases of the dispersion relation (7.3) determined by the signs $s_{1}$ and $s_{2}$ in the two-way Boussinesq equation (7.2)

When the coefficients are nonzero, the Boussinesq equation can be put into standard form. Scale the independent and dependent variables: $\tau=a T, \xi=b X$, and $U=\rho u$; then values of $a, b, \rho$ can be chosen so that the two-way Boussinesq equation becomes

$$
u_{\tau \tau}+s_{1} u_{\xi \xi}+\left(\frac{1}{2} u^{2}\right)_{\xi \xi}+s_{2} u \xi \xi \xi \xi=0, \quad s_{1}, s_{2}= \pm 1
$$

with

$$
s_{1}=\operatorname{sign}(\mu \nu) \text { and } s_{2}=\operatorname{sign}(\mu \mathscr{K}) .
$$

The sign $s_{1}$ determines whether the unfolding is into the elliptic region $\left(s_{1}=+1\right)$ or into the hyperbolic region $\left(s_{1}=-1\right.$, in which case all characteristics are hyperbolic). The sign $s_{2}$ indicates whether the resulting two-way Boussinesq equation is good $\left(s_{2}=+1\right)$ or bad $\left(s_{2}=-1\right)$. In the latter case, the initial value problem for the linearized system about $u=0$ is ill-posed, and small initial data with zero mean is therefore expected to saturate to form nonlinear structures. The ill-posedness in the case $s_{2}=-1$ can be seen by considering the linearization of (7.2) about the trivial solution and introducing a normal mode solution of the form $\mathrm{e}^{\mathrm{i}(\hat{k} \xi+\hat{\omega} \tau)}$. The dispersion relation associated with the normal mode is then

$$
\hat{\omega}^{2}=-s_{1} \hat{k}^{2}+s_{2} \hat{k}^{4}
$$

There are four cases depending on the signs $s_{1}$ and $s_{2}$, and they are shown in Fig. 2. The figure plots $\hat{\omega}^{2}$ against $\hat{k}^{2}$ and so $\hat{\omega}^{2}<0$ indicates linear instability of the trivial solution which in turn reflects linear instability of the basic travelling wave. 
When $s_{1}<0$ (the upper two cases in Fig. 2) then either an unstable band emerges at finite $\hat{k}$ when $s_{2}=-1$ or the Boussinesq equation is hyperbolic for all wavenumbers $\left(s_{2}=+1\right)$. When $s_{1}>0$ (lower two cases in Fig. 2$)$ then either a cutoff wave number emerges with re-stabilization at finite $\hat{k}$ (as in the lower right diagram with $s_{2}=+1$ ), or instability is further enhanced for all wavenumbers $\left(s_{1}=+1\right.$ and $\left.s_{2}=-1\right)$.

The simplest class of nonlinear solutions of (7.2) are travelling solitary wave solutions, for example,

$$
u(\xi, \tau)=\widehat{u}(\xi+\gamma \tau)
$$

which satisfies the ODE

$$
\left(\gamma^{2} \widehat{u}+s_{1} \widehat{u}+\frac{1}{2} \widehat{u}^{2}+s_{2} \widehat{u}^{\prime \prime}\right)^{\prime \prime}=0
$$

Integrating and taking the function of integration to be constant

$$
s_{2} \widehat{u}^{\prime \prime}+\left(s_{1}+\gamma^{2}\right) \widehat{u}+\frac{1}{2} \widehat{u}^{2}=h .
$$

The constant of integration $h$ is fixed by initial data or the value of $\widehat{u}$ at infinity. For appropriate parameter values, this planar ODE has a family of periodic solutions and a homoclinic orbit which represent periodic travelling waves and a solitary travelling wave solution of (7.2). The implication of these solutions is that the transition from elliptic to hyperbolic of a periodic travelling wave of the original system generates a coherent structure in the transition, which is represented by the above solitary wave. However, there is much more complexity generated at the transition. Hirota (1973) shows that there is a large family of $M$-soliton solutions to (7.2) as well, where $M$ can be any natural number. Further details especially in the case $M=2$ are given in Hirota (1973). Blow-up can occur in the two-way Boussinesq equation even in the case of the good Boussinesq equation (Turitsyn 1993). It is also generated by a Lagrangian, and has both a Hamiltonian and multisymplectic structure (e.g. Sect. 10 of Bridges and Derks 2001; Chen 2005).

\section{CNLS Wavetrains with Coalescing Characteristics}

To illustrate the nonlinear theory it is applied to the modulation of two-phase wavetrains of a coupled nonlinear Schrödinger (CNLS) equation. This example serves two purposes: firstly, it shows that the coalescence of characteristics is quite common and appears even in the simplest of examples, and secondly, it shows that computing the coefficients in the emergent two-way Boussinesq equation is elementary once the properties of the basic state are found.

The CNLS equation is a canonical example of a PDE generated by a Lagrangian with a toral symmetry, $\mathbb{T}^{2}=S^{1} \times S^{1}$. Indeed any finite number of NLS equations can be coupled together to generate a toral symmetry $\mathbb{T}^{N}$ for any natural number $N$, and 
they will have explicit $N$-phase wavetrains which are also relative equilibria. Here, attention is restricted to two coupled NLS equations in the form

$$
\begin{aligned}
& \mathrm{i} \frac{\partial \Psi_{1}}{\partial t}+\alpha_{1} \frac{\partial^{2} \Psi_{1}}{\partial x^{2}}+\left(\beta_{11}\left|\Psi_{1}\right|^{2}+\beta_{12}\left|\Psi_{2}\right|^{2}\right) \Psi_{1}=0 \\
& \mathrm{i} \frac{\partial \Psi_{2}}{\partial t}+\alpha_{2} \frac{\partial^{2} \Psi_{2}}{\partial x^{2}}+\left(\beta_{21}\left|\Psi_{1}\right|^{2}+\beta_{22}\left|\Psi_{2}\right|^{2}\right) \Psi_{2}=0
\end{aligned}
$$

where the coefficients $\alpha_{j}, \beta_{i j}, i, j=1,2$, are given real constants, with $\beta_{21}=\beta_{12}$. The functions $\Psi_{j}(x, t)$ are complex-valued and $\mathrm{i}^{2}=-1$.

Coupled NLS equations appear in a wide range of applications. Two applications that motivated this work are the coupled NLS equations that appear in the theory of water waves (e.g. Roskes 1976; Ablowitz and Horikis 2015; Degasperis et al. 2019), and in models for Bose-Einstein condensates (e.g. Salman and Berloff 2009; Kevrekidis and Frantzeskakis 2016). The PDE (8.1) is the Euler-Lagrange equation for

$$
\mathcal{L}(\Psi)=\int_{t_{1}}^{t_{2}} \int_{x_{1}}^{x_{2}} L\left(\Psi_{t}, \Psi_{x}, \Psi\right) \mathrm{d} x \mathrm{~d} t
$$

with $\Psi:=\left(\Psi_{1}, \Psi_{2}\right)$ and

$$
\begin{aligned}
L=\frac{\mathrm{i}}{2} & \left(\bar{\Psi}_{1}\left(\Psi_{1}\right)_{t}-\Psi_{1}\left(\bar{\Psi}_{1}\right)_{t}\right)+\frac{\mathrm{i}}{2}\left(\bar{\Psi}_{2}\left(\Psi_{2}\right)_{t}-\Psi_{2}\left(\bar{\Psi}_{2}\right)_{t}\right) \\
& -\alpha_{1}\left|\left(\Psi_{1}\right)_{x}\right|^{2}-\alpha_{2}\left|\left(\Psi_{2}\right)_{x}\right|^{2}+\frac{1}{2} \beta_{11}\left|\Psi_{1}\right|^{4}+\beta_{12}\left|\Psi_{1}\right|^{2}\left|\Psi_{2}\right|^{2}+\frac{1}{2} \beta_{22}\left|\Psi_{2}\right|^{4}
\end{aligned}
$$

with the overline denoting complex conjugate.

The toral symmetry follows from the fact that $\left(\mathrm{e}^{\mathrm{i} \theta_{1}} \Psi_{1}, \mathrm{e}^{\mathrm{i} \theta_{2}} \Psi_{2}\right)$ is a solution of (8.1), for any $\left(\theta_{1}, \theta_{2}\right) \in S^{1} \times S^{1}$, when $\left(\Psi_{1}, \Psi_{2}\right)$ is a solution. The complex coordinates can be converted to real coordinates, generating a standard action of $\mathbb{T}^{2}$ but will not be needed as the main calculations can be done in the complex setting.

Noether's theorem gives the conservation laws

$$
\left(A_{j}\right)_{t}+\left(B_{j}\right)_{x}=0, \quad j=1,2,
$$

with

$$
A_{j}=\frac{1}{2}\left|\Psi_{j}\right|^{2} \quad \text { and } \quad B_{j}=\alpha_{1} \operatorname{Im}\left(\bar{\Psi}_{1}\left(\Psi_{1}\right)_{x}\right), \quad j=1,2
$$

The basic state is just the usual family of plane waves, but interpreted here as a family of relative equilibria associated with the $\mathbb{T}^{2}$ symmetry; it has the form,

$$
\Psi_{j}(x, t)=\Psi_{j}^{0}(\boldsymbol{\omega}, \mathbf{k}) \mathrm{e}^{\mathrm{i} \theta_{j}(x, t)}, \quad \theta_{j}(x, t)=k_{j} x+\omega_{j} t+\theta_{j}^{0}, \quad j=1,2 .
$$


Substitution into the governing equation (8.1) generates the required relationship between the amplitudes, frequencies and wavenumbers,

$$
\begin{aligned}
& \left|\Psi_{1}^{0}\right|^{2}=\frac{1}{\beta}\left(\beta_{22}\left(\omega_{1}+\alpha_{1} k_{1}^{2}\right)-\beta_{12}\left(\omega_{2}+\alpha_{2} k_{2}^{2}\right)\right) \\
& \left|\Psi_{2}^{0}\right|^{2}=\frac{1}{\beta}\left(\beta_{11}\left(\omega_{2}+\alpha_{2} k_{2}^{2}\right)-\beta_{21}\left(\omega_{1}+\alpha_{1} k_{1}^{2}\right)\right),
\end{aligned}
$$

with $\beta_{21}:=\beta_{12}$ and $\beta=\beta_{11} \beta_{22}-\beta_{12} \beta_{21} \neq 0$.

The key wave action vectors $\mathbf{A}(\boldsymbol{\omega}, \mathbf{k})$ and $\mathbf{B}(\boldsymbol{\omega}, \mathbf{k})$, needed for analysis of the linearization, are obtained by substituting (8.5) into the components of the conservation law (8.3),

$$
\mathbf{A}(\boldsymbol{\omega}, \mathbf{k}):=\left(\begin{array}{l}
\mathscr{A}_{1}(\boldsymbol{\omega}, \mathbf{k}) \\
\mathscr{A}_{2}(\boldsymbol{\omega}, \mathbf{k})
\end{array}\right)=\frac{1}{2 \beta}\left(\begin{array}{c}
\beta_{22}\left(\omega_{1}+\alpha_{1} k_{1}^{2}\right)-\beta_{12}\left(\omega_{2}+\alpha_{2} k_{2}^{2}\right) \\
\beta_{11}\left(\omega_{2}+\alpha_{2} k_{2}^{2}\right)-\beta_{21}\left(\omega_{1}+\alpha_{1} k_{1}^{2}\right)
\end{array}\right)
$$

and

$$
\mathbf{B}(\boldsymbol{\omega}, \mathbf{k}):=\left(\begin{array}{c}
\mathscr{B}_{1}(\boldsymbol{\omega}, \mathbf{k}) \\
\mathscr{B}_{2}(\boldsymbol{\omega}, \mathbf{k})
\end{array}\right)=\frac{\alpha_{1} k_{1}}{\beta}\left(\begin{array}{c}
\beta_{22}\left(\omega_{1}+\alpha_{1} k_{1}^{2}\right)-\beta_{12}\left(\omega_{2}+\alpha_{2} k_{2}^{2}\right) \\
\beta_{11}\left(\omega_{2}+\alpha_{2} k_{2}^{2}\right)-\beta_{21}\left(\omega_{1}+\alpha_{1} k_{1}^{2}\right)
\end{array}\right)
$$

The linear operator $\mathbf{E}(c)$ defined in (1.20) is

$$
\mathbf{E}(c):=\mathrm{D}_{\omega} \mathbf{A} c^{2}+\left(\mathrm{D}_{\mathbf{k}} \mathbf{A}+\mathrm{D}_{\omega} \mathbf{B}\right) c+\mathrm{D}_{\mathbf{k}} \mathbf{B},
$$

with

$$
\mathrm{D}_{\omega} \mathbf{A}=\frac{1}{2 \beta}\left(\begin{array}{cc}
\beta_{22} & -\beta_{12} \\
-\beta_{12} & \beta_{11}
\end{array}\right)
$$

and

$$
\mathrm{D}_{\mathbf{k}} \mathbf{A}=\frac{1}{\beta}\left(\begin{array}{cc}
\alpha_{1} \beta_{22} k_{1} & -\alpha_{2} \beta_{12} k_{2} \\
-\alpha_{1} \beta_{12} k_{1} & \alpha_{2} \beta_{11} k_{2}
\end{array}\right)=\mathrm{D}_{\omega} \mathbf{B}^{T}
$$

and

$$
\mathrm{D}_{\mathbf{k}} \mathbf{B}=\frac{1}{\beta}\left(\begin{array}{cc}
\alpha_{1} \beta\left|\Psi_{1}^{0}\right|^{2}+2 \beta_{22} \alpha_{1}^{2} k_{1}^{2} & -2 \beta_{12} \alpha_{1} \alpha_{2} k_{1} k_{2} \\
-2 \beta_{12} \alpha_{1} \alpha_{2} k_{1} k_{2} & \alpha_{2} \beta\left|\Psi_{2}^{0}\right|^{2}+2 \alpha_{2}^{2} \beta_{11} k_{2}^{2}
\end{array}\right)
$$

The characteristic polynomial is

$$
\Delta(c):=\operatorname{det}[\mathbf{E}(c)]=a_{0} c^{4}+a_{1} c^{3}+a_{2} c^{2}+a_{3} c+a_{4},
$$


with

$$
\begin{aligned}
& a_{0}=\frac{1}{4} \beta^{-1}, \\
& a_{1}=\beta^{-1}\left(\alpha_{1} k_{1}+\alpha_{2} k_{2}\right), \\
& a_{2}=\frac{1}{2} \beta^{-1}\left[\alpha_{1}\left(\beta_{11}\left|\Psi_{1}^{0}\right|^{2}+2 \alpha_{1} k_{1}^{2}\right)+\alpha_{2}\left(\beta_{22}\left|\Psi_{2}^{0}\right|^{2}+2 \alpha_{2} k_{2}^{2}\right)+8 \alpha_{1} \alpha_{2} k_{1} k_{2}\right], \\
& a_{3}=2 \alpha_{1} \alpha_{2} \beta^{-1}\left(k_{1}\left(\beta_{22}\left|\Psi_{2}^{0}\right|^{2}+2 \alpha_{2} k_{2}^{2}\right)+k_{2}\left(\beta_{11}\left|\Psi_{1}^{0}\right|^{2}+2 \alpha_{1} k_{1}^{2}\right)\right) \\
& a_{4}=\alpha_{1} \alpha_{2} \beta^{-1}\left(\left(\beta_{11}\left|\Psi_{1}^{0}\right|^{2}+2 \alpha_{1} k_{1}^{2}\right)\left(\beta_{22}\left|\Psi_{2}^{0}\right|^{2}+2 \alpha_{2} k_{2}^{2}\right)-\left|\Psi_{1}^{0}\right|^{2}\left|\Psi_{2}^{0}\right|^{2} \beta_{12}^{2}\right) .
\end{aligned}
$$

Coalescing characteristics are obtained by solving $\Delta(c)=\Delta^{\prime}(c)=0$ for $c$. This problem is solved numerically in Bridges and Ratliff (2019) by using the graphical sign characteristic. The function $\Delta(c)$ is plotted versus $c$ as parameters vary. That way roots and points where $\Delta^{\prime}(c)=0$ can be read off the graph. It is inspired by the graphical Krein signature introduced by Kollár and Miller (2014). Results in Bridges and Ratliff (2019) show that coalescing characteristics are plentiful in the Whitham modulation theory for CNLS.

According to the theory in this paper at coalescing characteristics, the following nonlinear modulation equation is generated

$$
\mu U_{T T}+\kappa\left(U U_{X}\right)_{X}+\mathscr{K} U_{X X X X}=0 .
$$

In principle, the quartic $\Delta(c)=0$ can be solved in closed form, but in practice this is lengthy and not illuminating, and numerical methods are more effective. For simplicity here, the case of Stokes waves (where $\mathbf{k}=0$ ) are considered, which restricts the parameter space significantly, and so calculations can be done explicitly. The strategy for calculating $\mu$ and $\kappa$ is to construct the averaged Lagrangian and use the formulas (1.28) and (2.25).

\subsection{Calculations for Stokes Waves}

Stokes waves are basic states corresponding to (8.4) but with $\mathbf{k}=0$, leading to the typical nonlinear Stokes frequency shift of the original near-linear wavepackets. With this restriction the coefficients $a_{1}$ and $a_{3}$ are identically zero reducing the coefficients in the polynomial in (8.12) to

$$
\begin{aligned}
& a_{0}=\frac{1}{4} \beta^{-1}, \\
& a_{2}=\frac{1}{2} \beta^{-1}\left[\alpha_{1} \beta_{11}\left|\Psi_{1}^{0}\right|^{2}+\alpha_{2} \beta_{22}\left|\Psi_{2}^{0}\right|^{2}\right], \\
& a_{4}=\alpha_{1} \alpha_{2}\left|\Psi_{1}^{0}\right|^{2}\left|\Psi_{2}^{0}\right|^{2} .
\end{aligned}
$$

There are four characteristics and they satisfy the biquadratic equation

$$
a_{0} c^{4}+a_{2} c^{2}+a_{4}=0
$$


giving

$$
\begin{aligned}
& c^{2}=-\alpha_{1} \beta_{11}\left|\Psi_{1}^{0}\right|^{2}-\alpha_{2} \beta_{22}\left|\Psi_{2}^{0}\right|^{2} \\
& \quad \pm \sqrt{\left(\alpha_{1} \beta_{11}\left|\Psi_{1}^{0}\right|^{2}-\alpha_{2} \beta_{22}\left|\Psi_{2}^{0}\right|^{2}\right)^{2}+4 \alpha_{1} \alpha_{2} \beta_{12}^{2}\left|\Psi_{1}^{0}\right|^{2}\left|\Psi_{2}^{0}\right|^{2}} .
\end{aligned}
$$

Coalescing characteristics occur precisely when the discriminant vanishes

$$
\left(\alpha_{1} \beta_{11}\left|\Psi_{1}^{0}\right|^{2}-\alpha_{2} \beta_{22}\left|\Psi_{2}^{0}\right|^{2}\right)^{2}+4 \alpha_{1} \alpha_{2} \beta_{12}^{2}\left|\Psi_{1}^{0}\right|^{2}\left|\Psi_{2}^{0}\right|^{2}=0 .
$$

One way to interpret this equation is as a line in the positive quadrant of $\left(\left|\Psi_{1}^{0}\right|^{2},\left|\Psi_{2}^{0}\right|^{2}\right)$ space defined by

$$
\alpha_{2} \beta_{22}^{2}\left|\Psi_{2}^{0}\right|^{2}=\alpha_{1}\left(\beta_{11} \beta_{22}-2 \beta_{12}^{2} \pm 2\left|\beta_{12}\right| \sqrt{-\beta}\right)\left|\Psi_{1}^{0}\right|^{2},
$$

which includes the conditions $\beta<0$ and $\alpha_{1} \alpha_{2}<0$ for reality. At coalescence, it follows from (8.14) that

$$
c_{g}^{2}=-\alpha_{1} \beta_{11}\left|\Psi_{1}^{0}\right|^{2}-\alpha_{2} \beta_{22}\left|\Psi_{2}^{0}\right|^{2},
$$

which carries with it the requirement that $\alpha_{1} \beta_{11}\left|\Psi_{1}^{0}\right|^{2}+\alpha_{2} \beta_{22}\left|\Psi_{2}^{0}\right|^{2}<0$, a condition that is effectively a generalization of the defocussing classification for the one-component NLS.

Now suppose parameters are such that $(8.15)$ is satisfied, and proceed to compute the required coefficients in (8.13). The eigenvector and generalized eigenvector of $\mathbf{E}\left(c_{g}\right)$ are,

$$
\begin{aligned}
& \zeta=\left(\begin{array}{c}
c_{g}^{2} \beta_{12} \\
\beta_{22} c_{g}^{2}+2 \alpha_{1} \beta\left|\Psi_{1}^{0}\right|^{2}
\end{array}\right), \\
& \boldsymbol{\gamma}=-\frac{8 c_{g} \alpha_{1} \beta_{12}\left|\Psi_{1}^{0}\right|^{2} \beta^{2}}{\beta_{22} c_{g}^{2}+2 \alpha_{1} \beta\left|\Psi_{1}^{0}\right|^{2}}\left(\begin{array}{l}
1 \\
0
\end{array}\right) .
\end{aligned}
$$

Now use these eigenvectors and the Jacobians (8.8), (8.9) and (8.10) to generate the coefficients of the emergent Boussinesq equation. The first computed is the coefficient of the time derivative term,

$$
\zeta^{T} \mathrm{D}_{\omega} \mathbf{A} \zeta+\zeta^{T} \mathbf{E}^{\prime}\left(c_{g}\right) \boldsymbol{\gamma}=4 c_{g}^{2} \kappa_{0}, \quad \text { with } \quad \kappa_{0}=4 \beta\left(\beta_{22} c_{g}^{2}+2 \alpha_{1} \beta\left|\Psi_{1}^{0}\right|^{2}\right) .
$$

Next, one may use the variation of the Lagrangian to show that the coefficient of the nonlinear term is

$$
\kappa=-\frac{3 c_{g}^{2} \kappa_{0}}{2\left|\Psi_{2}^{0}\right|^{2}}\left(\alpha_{1} \beta_{11}\left|\Psi_{1}^{0}\right|^{2}-\alpha_{2} \beta_{22}\left|\Psi_{2}^{0}\right|^{2}\right)\left(\alpha_{1} \beta_{11}\left|\Psi_{1}^{0}\right|^{2}-\alpha_{2} \beta_{22}\left|\Psi_{2}^{0}\right|^{2}+2 \alpha_{1} \beta_{12}\left|\Psi_{2}^{0}\right|^{2}\right),
$$

The coefficient of dispersion requires a Jordan chain analysis. This would require multisymplectification of CNLS and construction of the linear operator $\mathbf{L}$. However, 
this CNLS has been multisymplectified in Ratliff (2017a), where reduction to KdV and 2-parameter Boussinesq were studied, and the Jordan chain theory is close to this case. With minor modification of that analysis, the desired dispersive coefficient is found to be

$$
\zeta^{T} \mathbf{T}=\frac{\kappa_{0}\left(\alpha_{2}\left|\Psi_{1}^{0}\right|^{2}-\alpha_{1}\left|\Psi_{2}^{0}\right|^{2}\right)}{\left|\Psi_{1}^{0}\right|^{2}\left|\Psi_{2}^{0}\right|^{2}\left(\alpha_{1} \beta_{11}\left|\Psi_{1}^{0}\right|^{2}-\alpha_{2} \beta_{22}\left|\Psi_{2}^{0}\right|^{2}\right)}
$$

Each of these coefficients has a common factor $\kappa_{0}$, and so the two-way Boussinesq that emerges at the coalescence of characteristics simplifies to

$$
c_{g}^{2} U_{T T}+\left(\frac{1}{2} \tilde{\kappa} U^{2}+\tilde{\mathscr{K}} U_{X X}\right)_{X X}=0,
$$

with

$$
\begin{aligned}
\tilde{\kappa} & =-\frac{3 c_{g}^{2}}{8\left|\Psi_{2}\right|^{2}}\left(\alpha_{1} \beta_{11}\left|\Psi_{1}\right|^{2}-\alpha_{2} \beta_{22}\left|\Psi_{2}\right|^{2}\right)\left(\alpha_{1} \beta_{11}\left|\Psi_{1}\right|^{2}-\alpha_{2} \beta_{22}\left|\Psi_{2}\right|^{2}+2 \alpha_{1} \beta_{12}\left|\Psi_{2}\right|^{2}\right), \\
\tilde{\mathscr{K}} & =\frac{\alpha_{2}\left|\Psi_{1}^{0}\right|^{2}-\alpha_{1}\left|\Psi_{2}^{0}\right|^{2}}{4\left|\Psi_{1}^{0}\right|^{2}\left|\Psi_{2}^{0}\right|^{2}\left(\alpha_{1} \beta_{11}\left|\Psi_{1}^{0}\right|^{2}-\alpha_{2} \beta_{22}\left|\Psi_{2}^{0}\right|^{2}\right)} .
\end{aligned}
$$

With $\tilde{\kappa}$ and $\tilde{\mathscr{K}}$ nonzero, one can proceed to analyse the solutions of this equation using results in the literature (e.g. Hirota 1973; Turitsyn 1993). A detailed analysis of (8.17) and its implications for coupled NLS is outside the scope of this paper, but the diversity of complexity due to coalescing characteristics is clear; for example, evaluation of $\tilde{\mathscr{K}}$ along the lines (8.15) shows that (8.17) can be both positive (good Boussinesq) and negative (bad Boussinesq).

\section{Concluding Remarks}

This paper gives a complete weakly nonlinear theory for multiphase WMT when a pair of characteristics coalesce and transition from hyperbolic to elliptic. This transition, in the nonlinear problem creates nonlinear dispersive dynamics, and it transpires that the resulting normal form is the two-way Boussinesq equation. There are potential generalizations and new directions emerging from this theory.

\subsection{Generalization to $2+1$}

Although we have confined the discussion to $1+1$ dimensions, there is a natural generalization to $2+1$. A good starting point is the $2+1$ theory for the nonlinear modulation of single-phase wavetrains near coalescing characteristics (Bridges and Ratliff 2018). However, the Jordan chain theory in (6.5) will literally take on a new dimension, bringing in the intertwinement of three symplectic Jordan chains. On the other hand, key features like the frame speed, scaling, sign characteristic and reduction should carry over with appropriate modification. 


\subsection{Examples}

The results in this paper are universal, and are operational whenever a Lagrangian system has a suitable characteristic collision, which can be identified via the sign characteristic diagnostic used in Bridges and Ratliff (2019) and in this paper. There are examples in the literature where multiphase Whitham modulation theory has been applied and coalescing characteristics observed, and so the application of the theory in this paper is relevant. Two examples are Stokes travelling waves coupled to mean flow (Whitham 1967; Willebrand 1975) and modulation of viscous conduit periodic waves (Maiden and Hoefer 2016). Both of these examples have special features which require additional methodology. In the case of viscous conduit waves (Maiden and Hoefer 2016) the equations are not generated by a Lagrangian so the theory would have to be built on averaging of conservation laws. However, at coalescing characteristics one expects a two-way Boussinesq equation to be generated or analogous equation with additional non-conservative terms. The case of modulation of Stokes waves in shallow water (Whitham 1967) involves the full water wave problem and so the class of PDEs (2.3) has to be modified to account for the vertical variation of water wave fields. However, the full water wave problem has a multisymplectic structure (e.g. Chapter 14 of Bridges 2017) and so the theory should go through as in this paper, with appropriate modification.

\subsection{Larger Kernel of $\mathrm{E}\left(c_{g}\right)$}

In this paper, the basic state has $N$-phases but the dimension of the kernel of the $N \times N$ matrix $\mathbf{E}\left(c_{g}\right)$ is one. A different problem arises when the kernel of $\mathbf{E}\left(c_{g}\right)$ has dimension greater than one. In this case, the secondary reduction to $\operatorname{span}\{\zeta\}$ would be modified to $\operatorname{span}\left\{\zeta_{1}, \ldots, \zeta_{k}\right\}$ where $k \leq N$ is the dimension of the kernel of $\mathbf{E}\left(c_{g}\right)$. Then, $k$-additional coupled modulation equations are generated (one linked to each kernel direction).

\subsection{Moving Frames}

Whitham theory can also be formulated relative to any moving frame, and some frame speeds are more interesting than others. Ratliff (2019) shows that even generic Whitham theory, in the hyperbolic case, re-modulated relative to the appropriate characteristic frame, generates dispersion, on a longer time scale.

\subsection{Higher-Order Singularities}

Even in the case of two phases the parameter space is at least four dimensional, involving $\omega_{1}, \omega_{2}, k_{1}, k_{2}$, with further degrees of freedom emerging when system parameters are present. Hence, higher-order singularities are to be expected, e.g. more than two characteristics coalescing, or the coefficients $\mu, \kappa$, and $\mathscr{K}$ passing through zero. A 
potential rescaling and re-modulation could then be implemented leading to (as yet unknown) modulation equations replacing the two-way Boussinesq equation.

\subsection{Hyperbolicity of Multiphase Modulation}

For quadratic Hermitian matrix pencils a general condition for hyperbolicity can be given. Hyperbolicity meaning all real characteristics. Consider the $N$-phase case for

$$
\mathbf{E}(c) \mathbf{u}=0 \text { with } \mathbf{E}(c)=\mathrm{D}_{\omega} \mathbf{A} c^{2}+c\left(\mathrm{D}_{\omega} \mathbf{B}+\mathrm{D}_{\mathbf{k}} \mathbf{A}\right) c+\mathrm{D}_{\mathbf{k}} \mathbf{B} .
$$

Let $\mathbf{u} \in \mathbb{C}^{N}$ be arbitrary and define

$$
\begin{aligned}
\alpha & =\left\langle\mathbf{u}, \mathrm{D}_{\omega} \mathrm{Au}\right\rangle \\
\beta & =\frac{1}{2}\left\langle\mathbf{u},\left(\mathrm{D}_{\omega} \mathbf{B}+\mathrm{D}_{\mathbf{k}} \mathbf{A}\right) \mathbf{u}\right\rangle \\
\gamma & =\left\langle\mathbf{u}, \mathrm{D}_{\mathbf{k}} \mathbf{B u}\right\rangle,
\end{aligned}
$$

with $\langle\cdot, \cdot\rangle$ an inner product on $\mathbb{C}^{N}$. Guo and Lancaster (2005) study quadratic eigenvalue problems in general and applying their definition to (9.1) gives the following.

Definition The quadratic Hermitian matrix pencil (9.1) is hyperbolic if $\beta^{2}>\alpha \gamma$ for all nonzero $\mathbf{u} \in \mathbb{C}^{n}$.

If this condition is satisfied then all the characteristics are real, and no coalescence can occur. It is expected that the absence of coalescence would be rare. The CNLS example shows coalescence to be quite common, already with $N=2$. For arbitrary $N$, the parameter space $(\boldsymbol{\omega}, \mathbf{k})$ has dimension $2 N$ and so there is a high probability of coalescence. On the other hand, the above definition is a useful starting point in the analysis of multiphase WMEs. In the paper Guo and Lancaster (2005), they go on to give a number of sufficient conditions, and an algorithm for testing hyperbolicity and computing all the eigenvalues. These algorithms may be helpful in the study of the characteristics of multiphase Whitham theory.

\subsection{Number of Phases Tending to Infinity}

There is a known case where multiphase Whitham equations are hyperbolic. The paper of Willebrand (1975) derives the multiphase WMEs and takes the limit $N \rightarrow \infty$ and argues that they are hyperbolic in this limit. The argument proceeds by formally constructing explicit expressions for the leading order nonlinear corrections. Small divisors and divergence are expected, but only the leading order terms are studied. When $N$ is small, "splitting of group velocity" is noted in the weakly nonlinear case, which is equivalent to what is called "coalescing characteristics" in this paper. The unfolding of this split group velocity may lead to instability. But WILLEBRAND argues that the splitting disappears as $N \rightarrow \infty$. In the context of this paper, the limit $N \rightarrow \infty$ would just replace the matrix pencil $\mathbf{E}(c)$ by an Hermitian operator pencil and so Willebrand's claim would be that $\mathbf{E}(c)$ in the case $N \rightarrow \infty$ is hyperbolic. It is important 
to keep in mind that this argument is for multiphase modulation of weakly nonlinear Stokes waves only, but is an intriguing example nevertheless.

Open Access This article is licensed under a Creative Commons Attribution 4.0 International License, which permits use, sharing, adaptation, distribution and reproduction in any medium or format, as long as you give appropriate credit to the original author(s) and the source, provide a link to the Creative Commons licence, and indicate if changes were made. The images or other third party material in this article are included in the article's Creative Commons licence, unless indicated otherwise in a credit line to the material. If material is not included in the article's Creative Commons licence and your intended use is not permitted by statutory regulation or exceeds the permitted use, you will need to obtain permission directly from the copyright holder. To view a copy of this licence, visit http://creativecommons.org/licenses/by/4.0/.

\section{References}

Ablowitz, M.J., Benney, D.J.: The evolution of multi-phase modes for nonlinear dispersive waves. Stud. Appl. Math. 49, 225-238 (1970)

Ablowitz, M.J., Horikis, T.P.: Interacting nonlinear wave envelopes and rogue wave formation in deep water. Phys. Fluids 27, 012107 (2015)

Binding, P., Volkmer, H.: Eigencurves for two-parameter Sturm-Liouville equations. SIAM Rev. 38, 27-48 (1996)

Bridges, T.J.: Symmetry, Phase Modulation, and Nonlinear Waves. Cambridge University Press, Cambridge (2017)

Bridges, T.J.: A universal form for the emergence of the Korteweg-de Vries equation. Proc. R. Soc. A 469, 20120707 (2013)

Bridges, T.J., Derks, G.: The symplectic Evans matrix, and the instability of solitary waves and fronts. Arch. Rat. Mech. Anal. 156, 1-87 (2001)

Bridges, T.J., Hydon, P.E., Lawson, J.K.: Multisymplectic structures and the variational bicomplex. Math. Proc. Camb. Philos. Soc. 148, 159-178 (2010)

Bridges, T.J., Kostianko, A., Schneider, G.: A proof of validity for multiphase Whitham modulation theory. Proc. R. Soc. Lond. A 476, 20200203 (2020). https://doi.org/10.1098/rspa.2020.0203

Bridges, T.J., Ratliff, D.J.: On the elliptic-hyperbolic transition in Whitham modulation theory. SIAM J. Appl. Math. 77, 1989-2011 (2017)

Bridges, T.J., Ratliff, D.J.: Nonlinear modulation near the Lighthill instability threshold in $2+1$ Whitham theory. Philos. Trans. R. Soc. Lond. A 376, 20170194 (2018)

Bridges, T.J., Ratliff, D.J.: Krein signature and Whitham modulation theory: the sign of characteristics and the "sign characteristic". Stud. Appl. Math. 142, 314-335 (2019)

Chen, J.-B.: Multisymplectic geometry, local conservation laws and fourier pseudospectral discretization for the "good" Boussinesq equation. Appl. Math. Comp. 161, 55-67 (2005)

Degasperis, A., Lombardo, S., Sommacal, M.: Rogue wave type solutions and spectra of coupled nonlinear Schrödinger equations. Fluids 4, 57-77 (2019)

El, G.A., Hoefer, M.A., Shearer, M.: Dispersive and diffusive-dispersive shock waves for nonconvex conservation laws. SIAM Rev. 59, 13-61 (2017)

Flashka, H., Forest, M.G., McLaughlin, D.W.: Multiphase averaging and the inverse spectral solution of the Korteweg-de Vries equation. Commun. Pure Appl. Math. 33, 739-784 (1980)

Gohberg, I., Lancaster, P., Rodman, L.: Spectral analysis of selfadjoint matrix polynomials. Ann. Math. 112, 33-71 (1980)

Gohberg, I., Lancaster, P., Rodman, L.: Indefinite Linear Algebra and Applications. Basel, Birkhäuser (2005)

Guo, C.-H., Lancaster, P.: Algorithms for hyperbolic quadratic eigenvalue problems. Math. Comp. 74, 1777-1791 (2005)

Hirota, R.: Exact N-soliton of the wave equation of long waves in shallow water and in nonlinear lattices. J. Math. Phys. 14, 810-814 (1973)

Howard, J.E.: Stability of Hamiltonian equilibria. Scholarpedia 8(10), 3627 (2013) 
Kevrekidis, P.G., Frantzeskakis, D.J.: Solitons in coupled nonlinear Schrödinger models: a survey of recent developments. Rev. Phys. 1, 140-153 (2016)

Kollár, R., Miller, P.D.: Graphical Krein signature and the Evans-Krein function. SIAM Rev. 56, 73-123 (2014)

Lighthill, M.J.: Some special cases treated by the Whitham theory. Proc. R. Soc. Lond. A 299, 28-53 (1967)

Magnus, J.R., Neudecker, H.: Matrix Differential Calculus. Wiley, London (1988)

Maiden, M.D., Hoefer, M.A.: Modulations of viscous fluid conduit periodic waves. Proc. R. Soc. Lond. A 472, 20120707 (2016)

Mehrmann, V., Noferini, V., Tisseur, F., Xu, H.: On the sign characteristics of Hermitian matrix polynomials. Linear Algebra Appl. 511, 328-364 (2016)

Olbers, D., Willebrand, J., Eden, C.: Ocean Dynamics. Springer, Berlin (2012)

Ratliff, D.J.: On the reduction of coupled NLS equations to nonlinear phase equations via modulation of a two-phase wavetrain. IMA J. Appl. Math. 82, 1151-1170 (2017)

Ratliff, D.J.: Phase dynamics of periodic wavetrains leading to the 5th order KP equation. Phys. D 353, 11-19 (2017)

Ratliff, D.J.: Double degeneracy in multiphase modulation and the emergence of the Boussinesq equation. Stud. Appl. Math. 140, 48-77 (2018)

Ratliff, D.J.: The modulation of multiple phases leading to the modified KdV equation. Chaos 28, 093117 (2018)

Ratliff, D.J.: Dispersive dynamics in the characteristic moving frame. Proc. R. Soc. Lond. A 475, 20180784 (2019)

Ratliff, D.J., Bridges, T.J.: Multiphase wavetrains, singular wave interactions and the emergence of the Korteweg-de Vries equation. Proc. R. Soc. Lond. A 472, 20160456 (2016)

Ratliff, D.J., Bridges, T.J.: Reduction to modified KdV and its KP-like generalization via phase modulation. Nonlinearity 31, 3794-3813 (2018)

Roskes, G.J.: Nonlinear multiphase deep-water wavetrains. Phys. Fluids 19, 1253-1254 (1976)

Salman, H., Berloff, N.G.: Condensation of classical nonlinear waves in a two-component system. Phys. D 238, 1482-1489 (2009)

Sprenger, P., Hoefer, M.A.: Shock waves in dispersive hydrodynamics with nonconvex dispersion. SIAM J. Appl. Math. 77, 26-50 (2017)

Tisseur, F., Meerbergen, K.: The quadratic eigenvalue problem. SIAM Rev. 43, 235-286 (2001)

Turitsyn, S.K.: Blow-up in the Boussinesq equation. Phys. Rev. E 47, R796-R799 (1993)

van der Meer, J.C.: The Hamiltonian Hopf Bifurcation, Lect. Notes in Math. 1160. Springer, Berlin (1985)

Whitham, G.B.: A general approach to linear and nonlinear dispersive waves using a Lagrangian. J. Fluid Mech. 22, 273-283 (1965)

Whitham, G.B.: Non-linear dispersion of water waves. J. Fluid Mech. 27, 399-412 (1967)

Whitham, G.B.: Linear and Nonlinear Waves. Wiley, New York (1974)

Willebrand, J.: Energy transport in a nonlinear and inhomogeneous random gravity wave field. J. Fluid Mech. 70, 113-126 (1975)

Williamson, J.: On the algebraic problem concerning the normal forms of linear dynamical systems. Am. J. Math. 58, 141-163 (1936)

Publisher's Note Springer Nature remains neutral with regard to jurisdictional claims in published maps and institutional affiliations. 